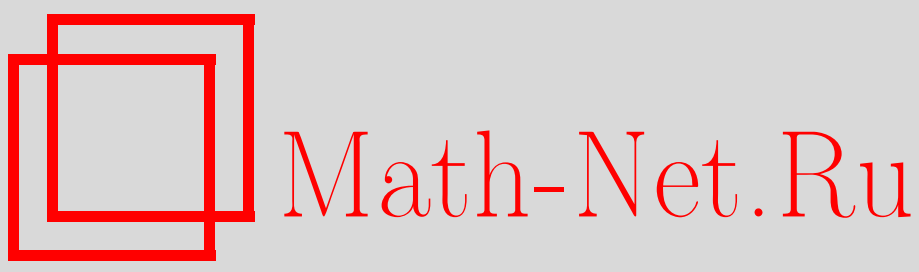

О. Н. Агеев, Функция кратности спектра и геометрические представления перекладываний, Матем. сб., 1999, том 190, номер 1, 3-28

DOI: https://doi.org/10.4213/sm376

Использование Общероссийского математического портала Math-Net.Ru подразумевает, что вы прочитали и согласны с пользовательским соглашением

http://www . mathnet.ru/rus/agreement

Параметры загрузки:

IP : 54.198 .67 .100

26 апреля 2023 г., $17: 02: 23$ 
УДК 517.9

\title{
О.Н. Агеев \\ Функция кратности спектра и геометрические представления перекладываний
}

\begin{abstract}
В статье решена задача о том, каким может быть множество $\mathscr{M}$ значений функции кратности спектра эргодического (строго эргодического) перекладывания, а также просто эргодической динамической системы. С помощью метода геометрических представлений построены типичные в метрическом и топологическом смысле подмножества преобразований в соответствующих подклассах с заранее заданным набором значений функции кратности спектра (естественно, из $\mathbb{N} \cup\{\infty\})$. В построенных классах преобразований присутствует новьй спектральный эффект - компонента спектра кратности 1 не совпадает со спектром любого фактора этих преобразований. Конструируются конкретные примеры строго эргодических перекладьваний со всеми возможными наборами значений функции кратности спектра.

Библиография: 21 название.
\end{abstract}

В статье рассматриваются сохраняющие непрерывную меру $\mu$ преобразования $T$ пространства Лебега $(X, \mu), \mu(X)=1$, называемые динамическими системами, и индуцированные ими унитарные операторы на $L_{2}(\mu):(T f)(x)=f(T x)$. Преобразование $T$ множества $X$ и соответствующий ему оператор часто называют автоморфизмами и обозначают одним и тем же символом. В статье рассматриваются множества $\mathscr{M}(T)$ всех значений функции кратности спектра унитарного оператора, отвечающего преобразованию $T$ в разных подклассах.

Настоящая работа относится к циклу вопросов, ставших классическими в теории динамических систем. Интенсивное исследование (см. обзоры [1], [2]) спектров унитарных операторов, отвечающих указанным преобразованиям, начавшееся после работы фон Неймана (в [3] было доказано, что спектральный инвариант является полным в классе преобразований с точечным спектром), выкристаллизовало сложность одной из основных нерешенных проблем теории преобразований с инвариантной мерой: определить точные условия на спектр унитарного оператора, при которых он может быть реализован динамической системой (см. [4], [5]). Не решены даже частные случаи этой задачи: существует ли преобразование с простьм лебеговским спектром (проблема Банаха) и даже с однократной лебеговской компонентой. Заметим, что примеры преобразований с лебеговской компонентой любой четной кратности построены в [6], [7].

Как известно, спектр унитарного оператора полностью определяется следующей парой: функцией кратности спектра и мерой максимального спектрального типа. В классических подклассах ( $K$-автоморфизмы, гауссовские динамические системы, автоморфизмы с положительной энтропией или с чисто точечньм спектром) эргодические преобразования имеют либо простой спектр, либо неограниченную функцию кратности спектра, см. [8].

Работа выпо лнена при частичной финансовой поддержке Российской программы поддержки ведущих научных школ (грант № 96-15-96135) и фонда INTAS-RFBR (грант № 95-418). 
В работе Оселедца [9] построены первые примеры автоморфизмов (являющихся перекладываниями) с конечной, отличной от единицы функцией кратности спектра (здесь наибольший элемент множества $\mathscr{M}(T)$ заключен в промежутке от 2 до 30). Робинсоном [10] найдены примеры преобразований $T$ с $\mathscr{M}(T)=\{1, n\}$ для любого натурального $n$ (см. также работу [11]) и эти преобразования реализованы в перекладываниях. В работах [12], [13] последовательно уточнялся класс подмножеств из $\mathbb{N}$, допускающих реализацию как $\mathscr{M}(T)$ для эргодических преобразований $T$. Заметим, что эти конструкции существенно использовали наличие в централизаторе $T$ косого произведения с непрерывным групповым автоморфизмом, даюшее дополнительное ограничение на $\mathscr{M}(T)$ следующего вида: $m_{1}, m_{2} \in \mathscr{M}(T) \Rightarrow \operatorname{HOK}\left(m_{1}, m_{2}\right) \in \mathscr{M}(T)$. Кроме того, среди преобразований с такого типа $\mathscr{M}(T)$ доказано сушествование аналитических диффеоморфизмов конечномерного тора, см. [14].

В настоящей работе предложен другой подход, основанньй на геометрических моделях (см. [15], [16]). Этот метод не использует групповые автоморфизмы и обладает конструктивньм потенциалом. K примеру, с помощью предложенной надстройки (см. (2)) можно с легкостью добавлять столько значений для функции кратности, сколько потребуется. В частности, можно надстроить преобразование с непростым конечнократным спектром так, что при этом спектр дополнится только циклическим пространством.

Отметим, что среди строящихся ниже автоморфизмов $T_{\omega_{\beta}}$ нет метрически изоморфных преобразованиям из вьшеупомянутых работ, исключая, возможно, тривиальные случаи $\mathscr{M}\left(T_{\omega_{\beta}}\right)=\{1\}, \mathscr{M}\left(T_{\omega_{\beta}}\right)=\{1,2\}$, так как компонента спектра кратности 1 не совпадает со спектром любого фактора этих автоморфизмов (в отличие от конструкций в [9]-[14]). Действительно, последнее утверждение есть непосредственное следствие того обстоятельства, что произведения ограниченных функций из этой компоненты не всегда будут принадлежать этой компоненте. Таким образом, в предложенной конструкции наблюдается новый спектральный эффект.

Пусть заданы полуинтервал $X=[a, b)$ и точки $a_{1}, a_{2}, \ldots, a_{m}, a<a_{1}<\cdots<$ $a_{m}<b$. Перекладыванием называется преобразование, являюшееся сдвигом на каждом из полуинтервалов $\left[a, a_{1}\right),\left[a_{1}, a_{2}\right), \ldots,\left[a_{m}, b\right)$. Обычно фиксируют набор длин этих полуинтервалов $\lambda=\left(\lambda_{1}, \lambda_{2}, \ldots, \lambda_{m+1}\right) \in \mathbb{R}^{m+1}$ и перестановку $\pi$, полностью определяющие перекладывание $T_{\lambda, \pi}$. Хорошо известно, что перекладывания не являются перемешиваюшими, имеют конечный ранг и, следовательно, ограниченную функцию кратности спектра. Этим, по-видимому, исчерпьвается информация о спектральных свойствах общих перекладьваний. В работе Вича [17] доказано, что в классе всех перекладываний $T_{\lambda, \pi}$ с фиксированной неприводимой перестановкой $\pi$ содержится множество полной меры Лебега по $\lambda$ перекладываний $T_{\lambda, \pi}$ с $\mathscr{M}\left(T_{\lambda, \pi}\right)=\{1\}$. Очевидно, что если $T$ эргодично, то $1 \in \mathscr{M}(T)$, где 1 отвечает спектральной мере с носителем в нуле и циклическому пространству $\left\{f \in L_{2}(\mu): T f=f\right\}$.

В настоящей работе для любого ограниченного $\mathscr{M} \subseteq \mathbb{N}, 1 \in \mathscr{M}$, построено множество полной меры по $\beta$ строго эргодических преобразований $T_{\omega_{\beta}}$ с $\mathscr{M}\left(T_{\omega_{\beta}}\right)=\mathscr{M}$ в подклассе перекладываний. Кроме того, для любого $\mathscr{M} \subseteq \mathbb{N} \cup\{\infty\}, 1 \in \mathscr{M}$, построено типичное множество (см. теорему 1) в подклассе эргодических преобразований $T$ пространства Лебега с $\mathscr{M}(T)=\mathscr{M}$. Соответствующий факт о типичности в слабой топологии эргодических преобразований пространства Лебега с $\mathscr{M}(T)=\{1\}$ хорошо известен. Эти преобразования имеют сингулярньй спектр, 
тем самым проблема Банаха имеет отрицательное решение в рассматриваемом классе.

Заметим, что принято рассматривать спектр преобразований в чуть меньшем инвариантном подпространстве $\left\{f \in L_{2}(\mu): f=\text { const }\right\}^{\perp}$. В значительной мере интерес здесь обусловлен нерешенной проблемой Рохлина - сушествует ли эргодическое преобразование с однородньм конечнократным спектром, отличньпм от простого, т.е. с $\mathscr{M}(T)=\{n\}$, где $n \notin\{1, \infty\}$ (см. [1], [10], [18]).

Перейдем к точньм формулировкам.

\section{§1. Конструкция преобразований и основные формулировки}

В статье мы используем следующие обозначения:

$$
\mathscr{M}=\left\{1, n_{1}, n_{2}, \ldots\right\} \subseteq \mathbb{N}, \quad \widetilde{N}=\sum_{j} n_{j}, \quad \tilde{N} \leqslant \infty,
$$

$\alpha$ - число из полуинтервала $[0,1)$, естественно отождествленного с окружностью $\mathbb{S}^{1} ; \beta$ - либо число из $\mathbb{S}^{1}$, либо $\beta=\left(\beta_{1}, \beta_{2}, \ldots\right) \in \mathbb{S}^{\widetilde{N}} ; T_{\alpha}$ - поворот в пространстве $([0,1), m): T_{\alpha} x=x+\alpha(\bmod 1) ; E-$ тождественное преобразование (или оператор); $H_{1} \oplus H_{2}$ - прямая сумма гильбертовых пространств $H_{i}$. Для преобразования $T$ пространства Лебега $(X, \mu), \mu(X)=1: C(h)=C_{T}(h)$ - наименьшее замкнутое, инвариантное относительно оператора $T$, содержащее вектор $h$ подпространство в $L_{2}(\mu)$ (называемое циклическим); $\sigma_{h}$ - спектральная мера, отвечающая вектору $h \in L_{2}(\mu)$, т.е. единственная борелевская мера на $\mathbb{S}^{1} \equiv[0,1)$ с моментами

$$
\widehat{\sigma}_{h}(n)=\int_{0}^{1} e^{2 \pi i n \lambda} d \sigma_{h}(\lambda)=\left(T^{n} h, h\right) .
$$

По спектральной теореме имеем равенство $L_{2}(\mu)=\bigoplus_{i} C\left(h_{i}\right)$, где либо $\sigma_{h_{i}} \sim \sigma_{h_{j}}$, либо $\sigma_{h_{i}} \perp \sigma_{h_{j}}$. Отсюда

$$
\mathscr{M}(T)=\left\{k_{1}, k_{2}, \ldots\right\}, \quad k_{i}=\#\left\{j: \sigma_{h_{i}} \sim \sigma_{h_{j}}\right\},
$$

где \# $A$ обозначает количество элементов в множестве $A(\# A \leqslant \infty)$.

1.1. Конструкция $T_{\omega_{\beta}}$. Фиксируем $\alpha \in[0,1)$ и $\beta=\left(\beta_{1}, \beta_{2}, \ldots\right) \in \mathbb{S}^{\tilde{N}}$. Далее для удобства будем использовать и другую нумерацию координат $\beta=$ $\left(\beta_{11}, \beta_{12}, \ldots, \beta_{1 n_{1}}, \beta_{21}, \ldots, \beta_{2 n_{2}}, \beta_{31}, \ldots\right)$ в $\mathbb{S}^{\widetilde{N}}$, учитывающую структуру множества $\mathscr{M}$. Определим сохраняюшее меру Хаара $\mu=m \times l \times l \times \cdots$ преобразование $T_{\omega_{\beta}}$, действуюшее в $\mathbb{S}^{1} \times \mathbb{Z}_{2}^{\widetilde{N}} \equiv[0,1) \times\{-1,1\}^{\widetilde{N}}$, по формуле

$$
T_{\omega_{\beta}}(x, y)=\left(T_{\alpha} x, \omega_{\beta}(x) * y\right),
$$

где

$$
\begin{gathered}
y=\left(y_{11}, y_{12}, \ldots\right), \quad y_{i j} \in\{-1,1\}, \\
\omega_{\beta}(x)=\left(\omega_{\beta_{11}}(x), \omega_{\beta_{11}}\left(x-\beta_{12}\right), \ldots, \omega_{\beta_{11}}\left(x-\beta_{1 n_{1}}\right), \omega_{\beta_{21}}(x), \omega_{\beta_{21}}\left(x-\beta_{22}\right), \ldots\right), \\
\quad \beta \in \mathbb{S}^{\widetilde{N}}, \\
\omega_{\gamma}(x)=\left\{\begin{array}{rr}
-1, & 0 \leqslant x<\gamma, \\
1, & \gamma \leqslant x<1,
\end{array}\right. \\
\omega_{\gamma_{1}}\left(x-\gamma_{2}\right) \equiv \omega_{\gamma_{1}}\left(T_{1-\gamma_{2}} x\right), \quad \mathbb{S}_{1}, \gamma_{2} \in \mathbb{S}^{1} \equiv[0,1),
\end{gathered}
$$


и “*” - покоординатное умножение в $\mathbb{Z}_{2}^{\widetilde{N}}$ (в зарубежной литературе более распространена запись $G$-расширения с аддитивной груповой операцией).

При $\widetilde{N}=1$ преобразования (2) были предложены фон Нейманом и долгое время служили источником разнообразных контрпримеров ко многим гипотезам эргодической теории (см. [19]).

1.2. Спектральные результаты. Ясно, что пространство $\mathbb{S}^{\widetilde{N}}$ является польским (полным метрическим сепарабельньм) в стандартной топологии покоординатной сходимости. Некоторое свойство называют типичнылм в группе $\mathbb{S}^{\widetilde{N}}$ (в топологическом смысле), если оно выполнено для всех $\beta$ из некоторого плотного подмножества типа $G_{\delta}$ в $\mathbb{S}^{\widetilde{N}}$. Элементы этого подмножества будем называть типичнымм. Имеет место следующий результат.

ТЕОрема 1. Для любого иррационального $\alpha$ и типичного $\beta \in \mathbb{S}^{\widetilde{N}}$ преобразования $T_{\omega_{\beta}}$ из (2) эргодичны и $\mathscr{M}\left(T_{\omega_{\beta}}\right)=\mathscr{M}$.

СлЕДСТВИЕ 1. Для любого унитарного оператора $U, 1 \in \mathscr{M}(U)$, существует эргодическая динамическая система $T$ с $\mathscr{M}(T)=\mathscr{M}(U)$.

СлЕДСТВИЕ 2. Для любого унитарного оператора $U, 1 \in \mathscr{M}(U)$, такого, что $\max \{n: n \in \mathscr{M}(U)\}<\infty$, существуют эргодические перекладывания $T$ $c \mathscr{M}(T)=\mathscr{M}(U)$.

(В действительности при $\widetilde{N}<\infty$ все $T_{\omega_{\beta}}$ суть перекладывания.)

Обозначим через $\Lambda_{d}(T)$ группу собственных значений преобразования $T$. Следующее замечание показывает типичность непрерывности спектра $T_{\omega_{\beta}}$ в дополнении к спектру $T_{\alpha}$.

ЗАмЕчаниЕ 1 . Для любого иррационального $\alpha$ и типичного $\beta \in \mathbb{S}^{\widetilde{N}}$ преобразования $T_{\omega_{\beta}}$ удовлетворяют дополнительному ограничению

$$
\Lambda_{d}\left(T_{\omega_{\beta}}\right)=\Lambda_{d}\left(T_{\alpha}\right) \equiv\left\{e^{2 \pi i k x}\right\}_{k \in \mathbb{Z}}
$$

Следующие утверждения показывают типичность в метрическом смысле преобразований $T$ с заданньп $\mathscr{M}(T)=\mathscr{M}$.

ЗАмечАниЕ 2. Для почти всех $\beta \in \mathbb{S}^{\widetilde{N}}$ по мере $m \times m \times \cdots$

$$
\mathscr{M}\left(T_{\omega_{\beta}}\right)=\mathscr{M}
$$

ЗАмечАниЕ 3 . В формулировке теоремы 1 условие эргодичности $T_{\omega_{\beta}}$ можно опустить, так как оно следует из того, что $1 \in \mathscr{M}\left(T_{\omega_{\beta}}\right)$.

ТЕорема 2. Для любого иррационального а и произвольного $\mathscr{M}$ из (1) если $\widetilde{N}<\infty$, то для почти всех $\beta \in \mathbb{S}^{\widetilde{N}}$ по мере $m \times m \times \cdots$ перекладывания $T_{\omega_{\beta}}$ из (2) строго әргодичньи и $\mathscr{M}\left(T_{\omega_{\beta}}\right)=\mathscr{M}$.

Заметим, что эргодичность относительно меры Лебега не гарантирует единственность, с точностью до константы, инвариантной борелевской меры, т.е. строгую эргодичность перекладьваний (см. [20]).

Далее в $\S 3$ вводится отношение эквивалентности на группе характеров $\widehat{\mathbb{Z}}_{2}^{\widetilde{N}}$ и приводится редукция теоремы $1 \mathrm{k}$ теореме 3 , формулируемой в терминах этой эквивалентности. Доказательства теорем 2,3 , следствий и замечаний проводятся 
в $\S 6$. Параграфы 2, 4, 5 содержат подготовительный материал, фактически сводящий (на основе метода геометрических моделей и индуцированных им метрик, см. $\S 2$, с использованием технических лемм, см. $\S 4)$ доказательство теоремы 3 к поиску центров окрестностей - точек $\beta(n) \in \mathbb{S}^{\widetilde{N}}$ в $n$-х моделях (см. $\S 5$ ) с нужными асимптотическими свойствами моментов.

Конструктивньй подход, используемый в доказательстве теоремы 1 (или 3), дает возможность построить в $\S 7$ конкретные элементы из типичного множества с $\mathscr{M}\left(T_{\omega_{\beta}}\right)=\mathscr{M}$ (см. предложение 5$)$.

Параграф 7 указывает также на любопытную связь геометрических моделей с одномерными отображениями.

\section{§2. Геометрические представления}

В [16] предложен метод геометрических моделей. Чтобы придать ясность и законченность изложению, уместно привести основные определения и обозначения.

2.1. Определения. Разложим число $\alpha \in \mathbb{S}^{1} \equiv[0,1)$ в непрерьвную дробь

$$
\alpha=\left[\alpha_{1}, \alpha_{2}, \ldots\right]=\frac{1}{\alpha_{1}+\frac{1}{\alpha_{2}+\cdots}},
$$

где $\alpha_{i}$ - натуральные числа. Напомним простейшие свойства непрерывных дробей. Представление $\alpha$ в виде (3) сушествует и единственно. Разложение (3) конечно, если $\alpha$ рационально, и бесконечно, если $\alpha$ иррационально. В первом случае

$$
\left[\alpha_{1}, \ldots, \alpha_{n}\right]=\frac{1}{\alpha_{1}+\frac{1}{\alpha_{2}+\cdots+\frac{1}{\alpha_{n}}}}=\frac{p_{n+1}}{q_{n+1}}
$$

где $p_{n}, q_{n}$ - взаимно простые числа. Числа $q_{n}$ можно определять из формул

$$
q_{0}=0, \quad q_{1}=1, \quad q_{n+1}=\alpha_{n} q_{n}+q_{n-1} .
$$

Справедлива следующая оценка

$$
\left|q_{n} \alpha-p_{n}\right|<\frac{1}{q_{n+1}} .
$$

Обозначим

$$
\frac{b_{n}}{a_{n}}=\frac{1}{\alpha_{n}+\frac{1}{\alpha_{n+1}+\cdots}}
$$

$\left(a_{n}, b_{n}-\right.$ меры верхних этажей в $n$-й модели). Введем последовательность $D(n) \subseteq \mathbb{R}^{2}, n=2,3, \ldots$ (см. рис. 1 ), по формуле

$$
\begin{gathered}
D(n)=\left[0,1+\frac{b_{n}}{a_{n}}\right) \times\left[0, \frac{q_{n-1}}{q_{n}}\right) \cup\left[c_{n}, c_{n}+1\right) \times\left[\frac{q_{n-1}}{q_{n}}, 1\right) \\
c_{n}=\frac{\left(1+(-1)^{n}\right) b_{n}}{2 a_{n}} .
\end{gathered}
$$



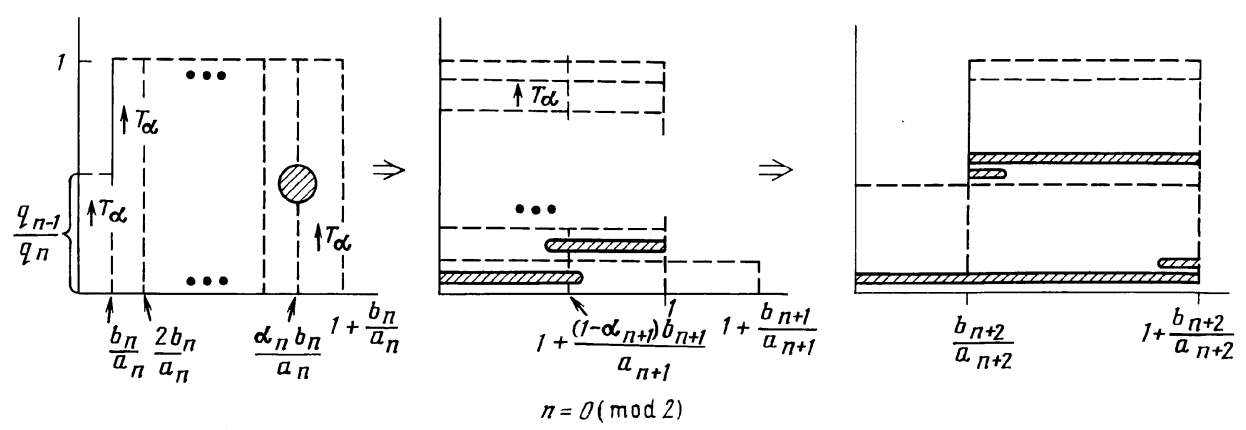

Рис. 1

Определим биекцию $\varphi_{1}:[0,1) \rightarrow D(2)$. Разобьем $[0,1)$ на полуинтервалы

$$
\begin{gathered}
\Delta_{0}(1)=\left[0,1-\alpha_{1} \alpha+\alpha\right), \\
\Delta_{k}(1)=\left[1+\left(k-\alpha_{1}\right) \alpha, 1+\left(k+1-\alpha_{1}\right) \alpha\right), \quad k=1,2, \ldots, \alpha_{1}-1 .
\end{gathered}
$$

Положим

$$
\varphi_{1}(x)=\left(z_{1}(2, x), z_{2}(2, x)\right)=\left(\frac{x}{\alpha}-k, \frac{k}{q_{2}}\right) \quad \text { при } x \in \Delta_{k}(1) .
$$

Определим биекции $\varphi_{n}: D(n) \rightarrow D(n+1)$ (см. рис. 1$)$. Если $n$ четно, то разобьем $\left[0,1+b_{n} / a_{n}\right)$ на полуинтервалы

$$
\Delta_{k}(n)=\left[k \frac{b_{n}}{a_{n}},(k+1) \frac{b_{n}}{a_{n}}\right), \quad k=0,1, \ldots, \alpha_{n}-1, \quad \Delta_{\alpha_{n}}(n)=\left[\alpha_{n} \frac{b_{n}}{a_{n}}, 1+\frac{b_{n}}{a_{n}}\right) .
$$

Положим

$$
\varphi_{n}\left(z_{1}, z_{2}\right)=\left(\frac{a_{n}}{b_{n}} z_{1}-k, \frac{q_{n}}{q_{n+1}}\left(z_{2}+\alpha_{n}-k\right)\right) \quad \text { при } z_{1} \in \Delta_{k}(n) .
$$

Если $n$ нечетно, то разобьем $\left[0,1+b_{n} / a_{n}\right)$ на полуинтервалы

$$
\begin{gathered}
\Delta_{0}(n)=\left[0,1+\left(1-\alpha_{n}\right) \frac{b_{n}}{a_{n}}\right), \\
\Delta_{k}(n)=\left[1+\left(k-\alpha_{n}\right) \frac{b_{n}}{a_{n}}, 1+\left(k+1-\alpha_{n}\right) \frac{b_{n}}{a_{n}}\right), \quad k=1,2, \ldots, \alpha_{n} .
\end{gathered}
$$

Положим

$$
\varphi_{n}\left(z_{1}, z_{2}\right)=\left(\frac{a_{n}}{b_{n}} z_{1}-k, \frac{q_{n}}{q_{n+1}}\left(z_{2}+k\right)\right) \text { при } z_{1} \in \Delta_{k}(n) .
$$

Множество $\varphi_{n-1} \varphi_{n-2} \ldots \varphi_{1}(A) \subseteq D(n)$ назовем $n$-й моделью $(n=2,3, \ldots)$ множества $A \subseteq \mathbb{S}^{1}$. Пусть $z_{1}(n, x), z_{2}(n, x)$ - координаты точки $x \in \mathbb{S}^{1}$ в $n$-й модели. Нетрудно убедиться в том, что:

1. $\left[0,1+b_{n} / a_{n}\right) \times\{0\} \subseteq \varphi_{n-1} \varphi_{n-2} \ldots \varphi_{1}\left(\mathbb{S}^{1}\right) ;$ 
2. коэффициент растяжения при переходе от полуинтервала $\Delta$ к набору полуинтервалов $\varphi_{n} \varphi_{n-1} \ldots \varphi_{1}(\Delta)$ не зависит от $\Delta$ (при фиксированном $n$ );

3. в $n$-й модели, обозначая через $P_{n}$ преобразование сдвига $\mathbb{R}^{2}$ вверх на $1 / q_{n}$, для $x \in D(n)$ имеем

$$
T_{\alpha} x=P_{n}(x)
$$

если $P_{n}(x) \in D(n)$,

$$
z_{2}\left(n, T_{\alpha} x\right)=0, \quad z_{1}\left(n, T_{\alpha} x\right)=z_{1}(n, x)+(-1)^{n+1} \frac{b_{n}}{a_{n}}\left(\bmod 1+\frac{b_{n}}{a_{n}}\right),
$$

если $P_{n}(x) \notin D(n)$.

2.2. Метрики $\rho_{n}$ и $\rho_{n}^{(k)}$, индуцированные геометрическими моделями. Фиксируем номер $n>1$. Рассмотрим дискретную подгруппу $\Gamma_{n} \subseteq \mathbb{R}^{2}$ следующего вида:

$$
\begin{aligned}
\Gamma_{n}=\left\{z=\left(z_{1}, z_{2}\right): \exists k, m \in \mathbb{Z}\right. \text { такие, что } \\
\qquad \begin{aligned}
z & \left.=k \cdot\left((-1)^{n+1}, \frac{q_{n-1}}{q_{n}}\right)+m \cdot\left((-1)^{n+1} \frac{b_{n}}{a_{n}},-1\right)\right\} .
\end{aligned}
\end{aligned}
$$

Евклидова метрика $\rho$ в $\mathbb{R}^{2}$ стандартным образом индуцирует метрику $\rho_{n}$ в факторгруппе $\mathbb{R}^{2} / \Gamma_{n}$, т.е. для $z^{\prime}, z^{\prime \prime} \in \mathbb{R}^{2} / \Gamma_{n}$ имеет место формула

$$
\rho_{n}\left(z^{\prime}, z^{\prime \prime}\right)=\min _{\substack{\tilde{z}^{\prime} \in z^{\prime} \Gamma_{n} \\ \tilde{z}^{\prime \prime} \in z^{\prime \prime} \Gamma_{n}}} \rho\left(\tilde{z}^{\prime}, \tilde{z}^{\prime \prime}\right)
$$

Нетрудно убедиться в том, что множество $D(n)$, введенное выше, есть фундаментальная область группы $\Gamma_{n}$, т.е. $\gamma_{1} D(n) \cap \gamma_{2} D(n)=\varnothing$ при $\gamma_{1}, \gamma_{2} \in \Gamma_{n}, \gamma_{1} \neq \gamma_{2}$, и $\bigcup_{\gamma \in \Gamma_{n}} \gamma D(n)=\mathbb{R}^{2} \cdot 1$

Естественное отождествление $D(n)$ с $\mathbb{R}^{2} / \Gamma_{n}$ определяет метрику $\rho_{n}$ на элементах $D(n)$. А отождествление в $n$-й модели окружности $\mathbb{S}^{1}$ с подмножеством $D(n)$ определяет метрику $\rho_{n}$ и на $\mathbb{S}^{1}$.

Сделаем следующие замечания:

1. Для любого $x \in \mathbb{S}^{1}$ множество $\left(T_{\alpha} x\right) \Gamma_{n} \subseteq \mathbb{R}^{2}$ получено сдвигом множества $x \Gamma_{n}$ вверх на $1 / q_{n}$.

2. Для любых $x \in \mathbb{S}^{1}$ и $n$

$$
\rho_{n}\left(x, T_{\alpha} x\right) \leqslant \frac{1}{q_{n}} .
$$

3. Метрика $\rho_{n}$ инвариантна относительно поворота на любой угол, т.е.

$$
\forall \beta \in \mathbb{S}^{1} \rho_{n}\left(T_{\beta} x, T_{\beta} y\right)=\rho_{n}(x, y) .
$$

Пункт 2 следует из 1 . Пункт 1 следует из определения $\Gamma_{n}$ и рассмотрения поворота на угол $\alpha$ в $n$-й модели. Для любого $k \in \mathbb{Z}, x, y \in \mathbb{S}^{1}$ в силу пункта 1 имеем $\rho_{n}\left(T_{\alpha}^{k} x, T_{\alpha}^{k} y\right)=\rho_{n}(x, y)$. Выберем $k$ из условия

$$
z_{2}\left(n, T_{\beta} x\right)=z_{2}\left(n, T_{\alpha}^{k} x\right)
$$

\footnotetext{
${ }^{1} \gamma A$ обозначает множество $\left\{z \in \mathbb{R}^{2}: \exists a \in A\right.$ такое, что $\left.\gamma+a=z\right\}$.
} 
Рассмотрим поворот на угол $\alpha_{0}$, где $T_{\alpha_{0}} T_{\alpha}^{k}=T_{\beta}$. В силу (7) для любого $x \in$ $\mathbb{S}^{1}$ множество $\left(T_{\alpha_{0}} x\right) \Gamma_{n}$ получено из $x \Gamma_{n}$ сдвигом по горизонтали. Поэтому для любых $x, y \in \mathbb{S}^{1}$ имеем $\rho_{n}\left(T_{\alpha_{0}} x, T_{\alpha_{0}} y\right)=\rho_{n}(x, y)$. Отсюда следует пункт 3 .

Для $\alpha, \beta \in \mathbb{S}^{\widetilde{N}}$ положим $\rho_{n}^{(k)}(\alpha, \beta)=\max _{1 \leqslant i \leqslant k} \rho_{n}\left(\alpha_{i}, \beta_{i}\right)$, где $\alpha=\left(\alpha_{1}, \alpha_{2}, \ldots\right)$, $\beta=\left(\beta_{1}, \beta_{2}, \ldots\right), \alpha_{i}, \beta_{i} \in \mathbb{S}^{1}$. Заметим, что полуметрика $\rho_{n}^{(k)}$ является метрикой в $\mathbb{S}^{\widetilde{N}} / \sim$, где $\alpha \sim \beta$ в точности тогда, когда $\alpha_{i}=\beta_{i}, i=1,2, \ldots, k$.

\section{§3. Эквивалентность характеров и редукция к теореме 3}

3.1. Характеры. Рассмотрим группу характеров $\widehat{\mathbb{Z}}_{2}^{\widetilde{N}}$ групшы $\mathbb{Z}_{2}^{\widetilde{N}} \equiv\{-1,1\}^{\widetilde{N}}$. Каждый характер $\chi(y)$ зависит только от конечного числа координат. Следовательно, для любого $y=\left(y_{11}, y_{12}, \ldots\right) \in\{-1,1\}^{\widetilde{N}}$ имеем $\chi(y)=y_{11}^{i_{11}} y_{12}^{i_{12}} \cdots$, где $i_{k l} \in\{0,1\}$ и $i_{k l}=0$ при достаточно большом $k$. Далее характер $\chi$ будем отождествлять с соответствующим набором $\left(i_{11}, i_{12}, \ldots\right)$ элементов $\{0,1\}^{\widetilde{N}}$ с нулевьм "хвостом".

Введем отношение эквивалентности на $\widehat{\mathbb{Z}}_{2}^{\widetilde{N}}$. Положим:

1. $\chi \sim \chi \forall \chi \in \widehat{\mathbb{Z}}_{2}^{\tilde{N}}$

2. если $\chi_{1} \neq \chi_{2}$, то

$$
\begin{gathered}
\chi_{1} \sim \chi_{2} \Leftrightarrow\left(i_{11}, i_{12}, \ldots\right) \sim\left(j_{11}, j_{12}, \ldots\right) \Leftrightarrow \exists k \exists m_{1}, m_{2} \leqslant n_{k} \\
\left(m_{1} \neq m_{2} \& i_{k m_{1}}=\sum_{r, l} i_{r l}=\sum_{r, l} j_{r l}=j_{k m_{2}}=1\right) .
\end{gathered}
$$

Гильбертово пространство $L_{2}\left(\mathbb{S}^{1} \times\{-1,1\}^{\widetilde{N}}, m \times l^{\widetilde{N}}\right)$ представимо в виде прямой суммы инвариантных относительно $T_{\omega_{\beta}}$ подпространств $H_{\chi}$, где $H_{\chi}=\left\{f(x) \chi(y): f(x) \in L_{2}\left(\mathbb{S}^{1}, m\right), \chi \in \widehat{\mathbb{Z}}_{2}^{\widetilde{N}}\right\}$. Оператор $T_{\omega_{\beta}}$ в подпространстве $H_{\chi}$ спектрально изоморфен оператору $\omega_{\chi}(x) T_{\alpha}: f(x) \mapsto \omega_{\chi}(x) \cdot f\left(T_{\alpha} x\right)$, действующему в $L_{2}\left(\mathbb{S}^{1}, m\right)$, где

$$
\omega_{\chi}(x) \equiv \omega_{\beta_{11}}^{i_{11}}(x) \omega_{\beta_{11}}^{i_{12}}\left(x-\beta_{12}\right) \cdots \omega_{\beta_{11}}^{i_{1 n_{1}}}\left(x-\beta_{1 n_{1}}\right) \omega_{\beta_{21}}^{i_{21}}(x) \omega_{\beta_{21}}^{i_{22}}\left(x-\beta_{22}\right) \cdots
$$

Обозначим: $V_{\chi}=\omega_{\chi}(x) T_{\alpha}, \sigma_{\chi}$ - мера максимального спектрального типа оператора $V_{\chi}$.

Доказательство следующей теоремы требует дополнительной подготовки (см. пп. $3.2,3.3, \S \S 4,5)$ и будет проведено в $\S 6$.

Теорема 3. Для любого иррационального $\alpha$ пространство $\mathbb{S}^{\tilde{N}}$ содержсит плотное подмножество типа $G_{\delta}$ таких $\beta$, что

1. при любом $\chi \in \widehat{\mathbb{Z}}_{2}^{\widetilde{N}}$ оператор $V_{\chi}$ имеет простой спектр;

2. $п р и л ю б ы x ~ \chi_{1}, \chi_{2} \in \widehat{\mathbb{Z}}_{2}^{\widetilde{N}}: \chi_{1} \sim \chi_{2} \Rightarrow \sigma_{\chi_{1}} \sim \sigma_{\chi_{2}}$;

3. $п р и л ю б ь х ~ \chi_{1}, \chi_{2} \in \widehat{\mathbb{Z}}_{2}^{\widetilde{N}}: \chi_{1} \nsim \chi_{2} \Rightarrow \sigma_{\chi_{1}} \perp \sigma_{\chi_{2}}$. 
ДоКАЗАТЕЛЬСТВо ТЕОРЕмЫ 1 . Перечислим мощности классов эквивалентности (8), (8') множества $\widehat{\mathbb{Z}}_{2}^{\widetilde{N}}: 1,1,1, \ldots, n_{1}, n_{2}, \ldots$ Пусть $\chi_{i}, i=1,2, \ldots,-$ характеры, лежащие в одноэлементных классах. В силу пा. 1,3 теоремы 3 гильбертово пространство $\bigoplus_{i} H_{\chi_{i}}$ является циклическим для оператора $T_{\omega_{\beta}}$ с циклическим вектором $h$, где

$$
\sigma_{h}=\sum_{k} 2^{-k} \sigma_{\chi_{k}}
$$

Учитьвая теорему 3 , получаем искомое представление $L_{2}\left(\mathbb{S}^{1} \times\{-1,1\}^{\widetilde{N}}\right.$, $m \times l^{\widetilde{N}}$ ) в виде прямой суммы циклических пространств.

Докажем эргодичность преобразования $T_{\omega_{\beta}}$. Предположим, что $T_{\omega_{\beta}}$ неэргодично. Тогда сушествуют такие $\chi \not \equiv \chi_{0} \equiv 1$ и $f \in H_{\chi}$, что $T_{\omega_{\beta}} f=f$. Поэтому $\sigma_{0} \leqslant \sigma_{\chi}$. Учитьвая $\sigma_{0} \leqslant \sigma_{\chi_{0}}$, получаем $\sigma_{\chi 0} \not \perp \sigma_{\chi}$. Это противоречит п. 3 теоремы 3. Доказательство теоремы 1 окончено.

3.2. Топологическая лемма. Выделим следующую топологическую лемму, фактически сводящую доказательство теоремы 3 к выбору необходимых окрестностей в $\mathbb{S}^{\widetilde{N}}$ в полуметрике $\rho_{n}^{(k)}$.

Фиксируем $\varepsilon>0, k \in \mathbb{N}, k \leqslant \widetilde{N}$, и последовательность $\beta_{n}, n=2,3, \ldots$, элементов множества $\mathbb{S}^{\widetilde{N}}$. Положим $U_{\varepsilon}(n)=\left\{\beta \in \mathbb{S}^{\widetilde{N}}: \rho_{n}^{(k)}(\beta, \beta(n))<\varepsilon\right\}$.

Лемма 1. Для любой последовательности номеров $n_{s}^{\prime}$ если $n_{s}^{\prime} \rightarrow \infty$ при $s \rightarrow \infty$, то множество $\bigcap_{m} \bigcup_{s>m} U_{\varepsilon}\left(n_{s}^{\prime}\right)$ есть плотное подмножсество типа $G_{\delta}$ пространства $\mathbb{S}^{\widetilde{N}}$ в стандартной топологии.

ДокаЗАтЕльСтво. Рассмотрим $n$-ю модель. Множество $\left\{x \in \mathbb{S}^{1}: \rho_{n}\left(x, x_{0}\right)<\varepsilon\right\}$ состоит из конечного числа интервалов и поэтому открыто в $\mathbb{S}^{1}$ в стандартной топологии. Множество $U_{\varepsilon}(n)$ есть пересечение конечного числа цилиндрических множеств вида

$$
U_{\varepsilon}(n, i, \beta(n))=\left\{\beta=\left(\beta_{1}, \beta_{2}, \ldots\right) \in \mathbb{S}^{\widetilde{N}}: \rho_{n}\left(\beta_{i}, \beta_{i}(n)\right)<\varepsilon\right\}
$$

и поэтому открыто в стандартной топологии.

Осталось показать, что для любых $n$ и $\varepsilon>0$ множество $\bigcup_{s>m} U_{\varepsilon}\left(n_{s}^{\prime}\right)$ всюду плотно в $\mathbb{S}^{\widetilde{N}}$. Фиксируем произвольную окрестность пространства $\mathbb{S}^{\widetilde{N}}$. Окрестность содержит некоторое цилиндрическое множество следующего вида:

$$
U\left(r, \Delta_{1}, \ldots, \Delta_{r}\right)=\left\{\beta=\left(\beta_{1}, \beta_{2}, \ldots\right) \in \mathbb{S}^{\tilde{N}}: \beta_{i} \in \Delta_{i}, i=1,2, \ldots, r\right\},
$$

где $\Delta_{i}$ - интервалы в $\mathbb{S}^{1}$. Положим $\varepsilon_{0}=\min _{i}\left|\Delta_{i}\right|$. В силу принципа Дирихле с учетом иррациональности $\alpha$ при некотором $n^{\prime \prime}$ множество $\{l \alpha(\bmod 1): l=$ $\left.0,1, \ldots, n^{\prime \prime}\right\}$ содержит хотя бы одну точку в каждом интервале $\Delta \in \mathbb{S}^{1} \equiv[0,1)$, где $|\Delta|=\varepsilon_{0}$. Выберем $s$ с условием

$$
\frac{n^{\prime \prime}}{q_{n_{s}^{\prime}}}<\varepsilon
$$

В силу выбора $n^{\prime \prime}$ для любого $i=1,2, \ldots, r$ существует $l_{i} \in\left\{0,1, \ldots, n^{\prime \prime}\right\}$ со свойством $\beta_{i}\left(n_{s}^{\prime}\right)+l_{i} \alpha(\bmod 1) \in \Delta_{i}$. Следовательно, имеем

$$
\begin{aligned}
\tilde{\beta}\left(n_{s}^{\prime}\right) & =\left(T_{\alpha}^{l_{1}} \beta_{1}\left(n_{s}^{\prime}\right), T_{\alpha}^{l_{2}} \beta_{2}\left(n_{s}^{\prime}\right), \ldots, T_{\alpha}^{l_{r}} \beta_{r}\left(n_{s}^{\prime}\right), \beta_{r+1}\left(n_{s}^{\prime}\right), \beta_{r+2}\left(n_{s}^{\prime}\right), \ldots\right) \\
& \in U\left(r, \Delta_{1}, \ldots, \Delta_{r}\right) .
\end{aligned}
$$


Для любого $i=1,2, \ldots, r$, комбинируя $(6),(9)$, получаем

$$
\rho_{n_{s}^{\prime}}\left(T_{\alpha}^{l_{i}} \beta_{i}\left(n_{s}^{\prime}\right), \beta_{i}\left(n_{s}^{\prime}\right)\right) \leqslant \frac{l_{i}}{q_{n_{s}^{\prime}}} \leqslant \frac{n^{\prime \prime}}{q_{n_{s}^{\prime}}}<\varepsilon .
$$

Поэтому $\tilde{\beta}\left(n_{s}^{\prime}\right) \in U_{\varepsilon}\left(n_{s}^{\prime}\right)$. Лемма 1 доказана.

3.3. Схема доказательства теоремы 3. Пункт 2 теоремы 3 следует из явного указания унитарного оператора $U$ со свойством $U V_{\chi_{1}}=V_{\chi_{2}} U$ для пары $\chi_{1}, \chi_{2}$, где $\chi_{1} \sim \chi_{2}$.

Для каждого характера $\chi \in \widehat{\mathbb{Z}}_{2}^{\tilde{N}}$ выбираются свои $\varepsilon>0$ и множества $U_{\varepsilon}(n)$. Доказательство простоты спектра оператора $V_{\chi}$ при $\beta \in \bigcap_{m} \bigcup_{s>m} U_{\varepsilon}\left(n_{s}^{\prime}\right)$ в целом следует доказательству теоремы 4 в [16] с учетом того, что распределение знаков $\omega_{\chi}(x)$ в $n$-й модели сводится к распределению знаков $\omega_{\beta_{i 1}}(x), \omega_{\beta_{i 1}+\beta_{i j}}(x)$ при $\beta_{i j} \in \mathbb{S}^{1}$.

Для каждой пары $\chi_{1}, \chi_{2}$ такой, что $\chi_{1} \nsim \chi_{2}$, также выбираются свои $\varepsilon>0$ и множества $U_{\varepsilon}(n)($ см. $\S 5)$. В доказательстве условия $\sigma_{\chi_{1}} \perp \sigma_{\chi_{2}}$ при $\beta \in$ $\bigcap_{m} \bigcup_{n>m} U_{\varepsilon}(n)$ используются следующие две леммы.

ЛЕмма 2. Для любого эргодического преобразования $T$ и функиии $\varphi \in L_{2}(X, \mu)$ такой, что $|\varphi|=1$ для почти всех $x$, если $T^{k_{i}}$ слабо сходится $\kappa$ E, то оператор $U_{\varphi}^{k_{i}}-\left(U_{\varphi}^{k_{i}} \mathbf{1}, \mathbf{1}\right) \cdot E$ слабо сходится $\kappa$ нулю, где $U_{\varphi}: f(x) \mapsto \varphi(x) f(T x)$ - унитарный оператор, действующий в $L_{2}(X, \mu)$.

Лемма 3. Пусть $\sigma_{j}$ - меры максимального спектрального типа унитарных операторов $U_{j}, j=1,2$, действующих в сепарабельном гильбертовом пространстве. Если для некоторых $c_{j}, j=1,2, c_{1} \neq c_{2}$, последовательности $U_{j}^{k_{m}}$ слабо сходятся $\kappa c_{j} E$ при $m \rightarrow \infty$, то $\sigma_{1} \perp \sigma_{2}$.

Добавив к леммам слабую сходимость $T_{\alpha}^{q_{n}}$ к $E$ при $n \rightarrow \infty$, для завершения доказательства теоремы 3 достаточно равномерно по $n$ отделить друг от друга пары $\left(V_{\chi_{1}}^{q_{n}} \mathbf{1}, \mathbf{1}\right),\left(V_{\chi_{2}}^{q_{n}} \mathbf{1}, \mathbf{1}\right)$, что, в свою очередь, достигается выбором $\beta(n)$ и $\varepsilon$ в силу равномерной по $n$ и $\beta=\left(\beta_{11}, \beta_{12}, \ldots\right)$ непрерывности в полуметрике $\rho_{n}^{(k)}$ функции $\left|\left(V_{\chi}^{q_{n}} \mathbf{1}, \mathbf{1}\right)\right|$ (см. лемму 6). Искомое плотное подмножество типа $G_{\delta}$ есть, очевидно, пересечение плотных подмножеств типа $G_{\delta}$, рассмотренных выше.

Далее будут использоваться следующие сокращения:

$$
\begin{gathered}
\omega_{\gamma}(x) \omega_{\gamma}\left(T_{\alpha} x\right) \cdots \omega_{\gamma}\left(T_{\alpha}^{q_{n}-1} x\right)=\omega_{\gamma}^{(n)}(x), \quad \gamma \in \mathbb{S}^{1} ; \\
\omega_{\chi}(x) \omega_{\chi}\left(T_{\alpha} x\right) \cdots \omega_{\chi}\left(T_{\alpha}^{q_{n}-1} x\right)=\omega_{\chi}^{(n)}(x), \quad \chi \in \widehat{\mathbb{Z}}_{2}^{\widetilde{N}} \\
\operatorname{Mom}_{n}(\chi, \beta)=\left|\left(V_{x}^{q_{n}} \mathbf{1}, \mathbf{1}\right)\right|=\left|\int_{\mathbb{S}^{1}} \omega_{\chi}^{(n)}(x) d m(x)\right| \\
=\left|\int_{\mathbb{S}^{1}} \omega_{\beta_{11}^{(n)^{i} 11}}^{(x) \cdot \omega_{\beta_{11}}^{(n)^{i} 12}}\left(x-\beta_{12}\right) \cdots d m(x)\right|
\end{gathered}
$$

Доказательство леммы 2 почти непосредственно следует из леммы 4.

Лемма 4 (см. [15]). Если последовательность функиий $h_{n}$ в сепарабельном гильбертовом пространстве ограничена по норме, $U$ - унитарный оператор 
$u h_{n}-U h_{n}$ слабо сходится $\kappa$ нулю, то $h_{n}-P_{U} h_{n}$, әде $P_{U}$ - оператор ортогонального проектирования на $H_{\mathrm{inv}}=\{h: h=U h\}$, слабо сходится к нулю.

Для $f, g \in L_{2}(X, \mu)$ и $\varphi_{i}(x)=\varphi(x) \varphi(T x) \cdots \varphi\left(T^{k_{i}-1} x\right)$, учитьвая соотношение $\left\|T \varphi_{i}-\varphi_{i}\right\|_{L_{2}} \rightarrow 0$ при $i \rightarrow \infty$ и равенство $H_{\mathrm{inv}}=\mathbb{C} \cdot \mathbf{1}$, имеем

$$
\left(\varphi_{i} \cdot f, g\right)-\left(\varphi_{i} \cdot \mathbf{1}, \mathbf{1}\right) \cdot(f, g) \rightarrow 0 \text { при } i \rightarrow \infty
$$

Учитывая, что $\left\|T^{k_{i}} f-f\right\|_{L_{2}} \rightarrow 0$ при $i \rightarrow \infty$ и что $\left(\varphi_{i} \cdot \mathbf{1}, \mathbf{1}\right)=\left(U_{\varphi}^{k_{i}} \mathbf{1}, \mathbf{1}\right)$, последовательно получаем

$$
\begin{aligned}
\left(U_{\varphi}^{k_{i}} f, g\right)-\left(\varphi_{i} f, g\right) \rightarrow 0 & \text { при } \quad i \rightarrow \infty, \\
\left(U_{\varphi}^{k_{i}} f, g\right)-\left(U_{\varphi}^{k_{i}} \mathbf{1}, \mathbf{1}\right) \cdot(f, g) \rightarrow 0 & \text { при } \quad i \rightarrow \infty .
\end{aligned}
$$

Лемма 2 доказана.

ДокАЗАТЕЛЬСтво леммы 3. Если меры $\sigma_{j}$ не взаимно сингулярны, то существует вероятностная борелевская мера $\rho$ на $\mathbb{S}^{1} \equiv[0,1)$, абсолютно непрерывная относительно $\sigma_{1}$ и $\sigma_{2}$. Учитывая, что для любого $f \in L_{2}\left(\mathbb{S}^{1}, \sigma_{j}\right)$ при $m \rightarrow \infty$

$$
\int_{\mathbb{S}^{1}} e^{2 \pi i k_{m} \lambda}|f|^{2} d \sigma_{j}(\lambda) \rightarrow c_{j} \int_{\mathbb{S}^{1}}|f|^{2} d \sigma_{j}(\lambda),
$$

при $m \rightarrow \infty$ для любого $j$ имеем

$$
\int_{\mathbb{S}^{1}} e^{2 \pi i k_{m} \lambda} d \rho(\lambda) \rightarrow c_{j}
$$

Полученное противоречие доказывает лемму 3.

\section{§4. Свойства моментов}

4.1. Далее нам потребуется распределение знаков функции $\omega_{\beta}^{(n)}(x)$ для точек $x$ в $n$-й модели $\left(\beta \in \mathbb{S}^{1}\right)$. Переформулируем с учетом $\S 2$ предложение 1 из [16] (см. рис. 2) в следующем виде:

ПрЕДЛОЖЕНИЕ 1. Множества $S_{ \pm}(n)=\left\{x: \omega_{\beta}^{(n)}(x)= \pm 1\right\}$ отделены друг от друга в п-й модели гранищей, являющейся образом линии

$$
\begin{aligned}
L_{n}=\{ & \left.\left(z_{1}, z_{2}\right) \in \mathbb{R}^{2}: z_{2}=z_{2}(n, \beta), z_{1} \in \Delta(n)\right\} \\
& \cup\left\{\left(z_{1}, z_{2}\right) \in \mathbb{R}^{2}: z_{1}=z_{1}(n, \beta), z_{2}(n, \beta)-1<z_{2} \leqslant z_{2}(n, \beta)\right\}
\end{aligned}
$$

при естественной проекции $\mathbb{R}^{2} \rightarrow \mathbb{R}^{2} / \Gamma_{n}$, әде $\left(z_{1}(n, x), z_{2}(n, x)\right)$ - координатьц точки $x \in \mathbb{S}^{1}$ в $n$-й модели и

$$
\Delta(n)= \begin{cases}{\left[z_{1}(n, \beta)-b_{n} / a_{n}, z_{1}(n, \beta)\right]} & \text { при нечетных } n \\ {\left[z_{1}(n, \beta), z_{1}(n, \beta)+b_{n} / a_{n}\right]} & \text { при четных } n\end{cases}
$$

Заметим, что мера одного этажа, т.е. $m\left\{x \in \mathbb{S}^{1}: z_{2}(n, x)=c(n)\right\}$, убывает экспоненциально по $n$, поэтому не будет влиять на последующие оценки, как, впрочем, и знак $\omega_{\beta}^{(n)}(x)$ на линии $L_{n}$.

\section{2. Две технические леммы.}



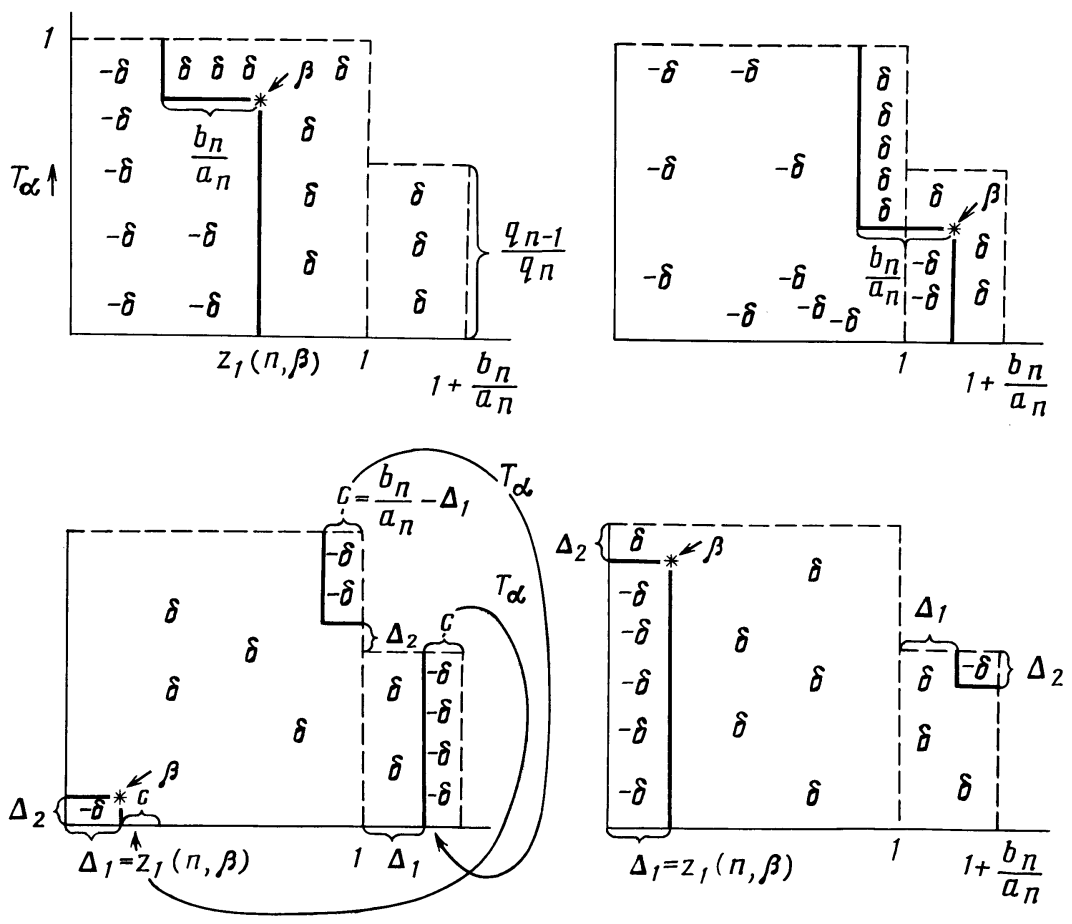

Рис. 2

Лемма 5. Существует такое число $\varepsilon>0$, что для любого номера $n>1$ найдутся $\beta_{i} \in \mathbb{S}^{1}, i=1,2, \ldots, 6$, удовлетворяющие следующим неравенствам:

$1.1-\left|\int_{\mathbb{S}^{1}} \omega_{\beta_{1}}^{(n)}(x) d m(x)\right|>\varepsilon$

$2.1-\left|\int_{\mathbb{S} 1} \omega_{\beta_{2}}^{(n)}(x) \omega_{\beta_{2}}^{(n)}\left(x-\beta_{3}\right) d m(x)\right|>\varepsilon$;

3. || $\int_{\mathbb{S}^{1}} \omega_{\beta_{4}}^{(n)}(x) \omega_{\beta_{4}}^{(n)}\left(x-\beta_{5}\right) \omega_{\beta_{4}}^{(n)}\left(x-\beta_{6}\right) d m(x)|-| \int_{\mathbb{S}^{1}} \omega_{\beta_{4}}^{(n)}(x) d m(x)||>\varepsilon$.

ДокАЗАТЕЛЬСТво. Пункт 1 сразу следует из предложения 1. Без ограничения общности будем рассматривать только нечетные $n$.

Докажем пункт 2. Фиксируем $\varepsilon^{\prime}=1 / 4$. Ясно, что в $n$-й модели для точки $0 \in \mathbb{S}^{1}$ имеем $z_{1}(n, 0)=0, z_{2}(n, 0)=\left(q_{n}-1\right) / q_{n}$. Выберем $\beta_{3}$ из условий $z_{1}\left(n, \beta_{3}\right)=\varepsilon^{\prime}$, $z_{2}\left(n, \beta_{3}\right)=z_{2}(n, 0)$, а $\beta_{2}$ - любая точка из $\mathbb{S}^{1}$ с условием

$$
\varepsilon^{\prime}+\frac{b_{n}}{a_{n}}<z_{1}\left(n, \beta_{2}\right)<1+\frac{b_{n}}{a_{n}}-\varepsilon^{\prime}
$$

(см. рис. 3). Тогда на $\mathbb{S}^{1} \equiv[0,1)$ имеем

$$
0<\beta_{3}<\beta_{2}<\beta_{2}+\beta_{3}<1 \text {. }
$$




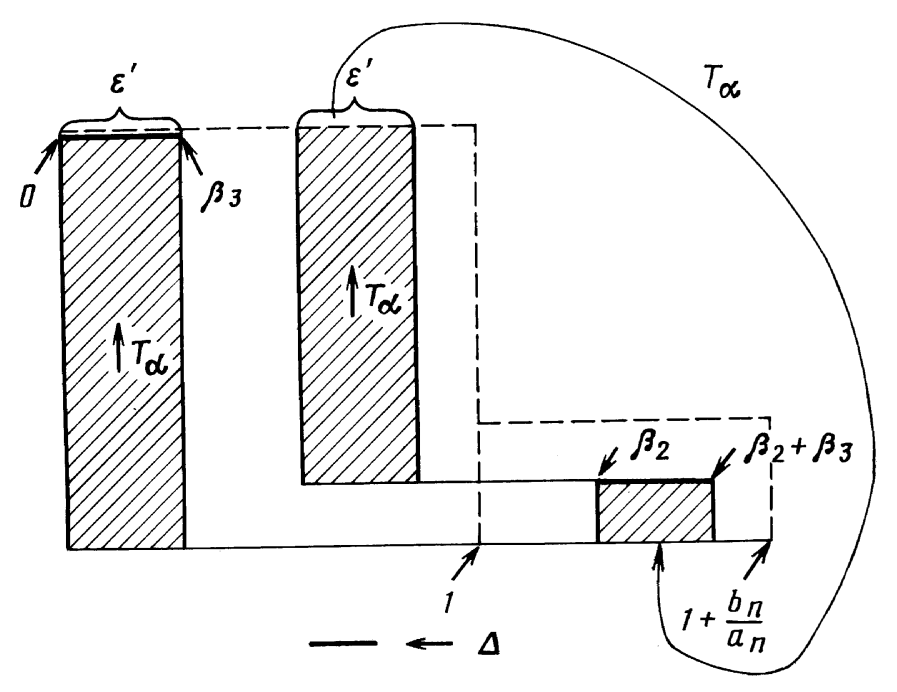

Рис. 3

Поэтому

$$
\omega_{\beta_{2}}(x) \omega_{\beta_{2}}\left(x-\beta_{3}\right)=-1 \Leftrightarrow x \in \Delta=\left[0, \beta_{3}\right) \cup\left[\beta_{2}, \beta_{2}+\beta_{3}\right) .
$$

В силу выбора $\beta_{2}, \beta_{3}$ (см. рис. 3) траектория длины $q_{n}$ любой точки (т.е. набор $\left.x, T_{\alpha} x, \ldots, T_{\alpha}^{q_{n}-1} x\right)$ попадает не более одного раза в множество $\Delta$. Поэтому имеем

$$
\begin{aligned}
m\{x & \left.\in \mathbb{S}^{1}: \omega_{\beta_{2}}^{(n)}(x) \omega_{\beta_{2}}^{(n)}\left(x-\beta_{3}\right)=-1\right\} \\
& =m\left\{x \in \mathbb{S}^{1}: \exists k \in\left\{0,1, \ldots, q_{n}-1\right\} \text { такое, что } T_{\alpha}^{k} x \in \Delta\right\}=q_{n} \cdot m(\Delta) \\
& =2 q_{n} \varepsilon^{\prime} \cdot m\left\{x \in \mathbb{S}^{1}: z_{2}(n, x)=z_{2}(n, 0), z_{1}(n, x) \leqslant 1\right\} \\
& =2 \varepsilon^{\prime} m\left\{x \in \mathbb{S}^{1}: z_{1}(n, x) \leqslant 1\right\} .
\end{aligned}
$$

Следовательно,

$$
\varepsilon^{\prime}<m\left\{x \in \mathbb{S}^{1}: \omega_{\beta_{2}}^{(n)}(x) \omega_{\beta_{2}}^{(n)}\left(x-\beta_{3}\right)=-1\right\}<2 \varepsilon^{\prime} .
$$

И, окончательно, имеем оценку (11), где $\varepsilon=1 / 2$.

Докажем пункт 3. Фиксируем $\varepsilon^{\prime}=1 / 6$. Выбираем $\beta_{4}, \beta_{5}, \beta_{6}$ из условий $z_{2}\left(n, \beta_{4}\right)=z_{2}\left(n, \beta_{5}\right)=z_{2}\left(n, \beta_{6}\right)=z_{2}(n, 0), z_{1}\left(n, \beta_{4}\right)=z_{1}\left(n, \beta_{5}\right)=\varepsilon^{\prime}$ и $z_{1}\left(n, \beta_{6}\right)=2 \varepsilon^{\prime}$. Тогда на $\mathbb{S}^{1} \equiv[0,1)$ имеем

$$
0<\beta_{4}=\beta_{5}<\beta_{6}=2 \beta_{4}<\beta_{6}+\beta_{4}<\frac{1}{q_{n}} .
$$

Поэтому

$$
\omega_{\beta_{4}}(x) \omega_{\beta_{4}}\left(x-\beta_{5}\right) \omega_{\beta_{4}}\left(x-\beta_{6}\right)=-1 \Leftrightarrow x \in\left[0, \beta_{6}+\beta_{4}\right) .
$$

Аналогично доказательству пункта 2 получим

$$
\begin{aligned}
& m\left\{x \in \mathbb{S}^{1}: \omega_{\beta_{4}}^{(n)}(x) \omega_{\beta_{4}}^{(n)}\left(x-\beta_{5}\right) \omega_{\beta_{4}}^{(n)}\left(x-\beta_{6}\right)=-1\right\} \\
& \quad=3 \varepsilon^{\prime} m\left\{x \in \mathbb{S}^{1}: z_{1}(n, x) \leqslant 1\right\}=3 m\left\{x \in \mathbb{S}^{1}: \omega_{\beta_{4}}^{(n)}(x)=-1\right\} .
\end{aligned}
$$


Следовательно, левая часть в (12) есть в точности

$$
4 \varepsilon^{\prime} m\left\{x \in \mathbb{S}^{1}: z_{1}(n, x) \leqslant 1\right\} .
$$

И, окончательно, имеем оценку (12), где $\varepsilon=1 / 3$.

Фиксируем характер $\chi$. Выбираем $k$ из следующего условия: функция $\chi$ зависит только от первых $k$ координат пространства $\{-1,1\}^{\widetilde{N}}$.

Лемма 6. Функиия $\operatorname{Mom}_{n}(\chi, \beta)$ равномерно непрерывна по $\beta, n$ относительно $\rho_{n}^{(k)}$, m.e. $\forall \varepsilon>0 \exists \delta>0 \forall n \forall \beta^{(1)}, \beta^{(2)} \in \mathbb{S}^{\tilde{N}}$

$$
\rho_{n}^{(k)}\left(\beta^{(1)}, \beta^{(2)}\right)<\delta \Rightarrow\left|\operatorname{Mom}_{n}\left(\chi, \beta^{(1)}\right)-\operatorname{Mom}_{n}\left(\chi, \beta^{(2)}\right)\right|<\varepsilon,
$$

әде $\beta^{(i)}=\left(\beta_{11}^{(i)}, \beta_{12}^{(i)}, \ldots\right), \beta_{j k}^{(i)} \in \mathbb{S}^{1}, i=1,2$.

Доказательство леммы 6 использует следующее предложение.

ПРЕДЛОЖЕНИЕ 2. Для любого $\varepsilon^{\prime}>0$ существует такое $\delta>0$, что для любого п выполняется

$$
\rho_{n}\left(\beta_{1}, \beta_{2}\right)<\delta \Rightarrow \exists i: m\left\{x \in \mathbb{S}^{1}: \omega_{\beta_{1}}^{(n)}(x) \neq(-1)^{(i)} \omega_{\beta_{2}}^{(n)}(x)\right\}<\varepsilon^{\prime} .
$$

ДокАЗАтЕльство. Условие $\rho_{n}\left(\beta_{1}, \beta_{2}\right)<\delta$ геометрически эквивалентно требованию: множество $\beta_{2} \Gamma_{n} \subset \mathbb{R}^{2}$ есть образ $\beta_{1} \Gamma_{n}$ при сдвиге на вектор $\bar{a}$, где $|\bar{a}|<\delta$. Ясно, что достаточно ограничиться следующими двумя случаями, в которых для определенности $n$ нечетно.

1. Сдвиг по горизонтали на вектор $\bar{a}$. Выберем $z^{\prime}=\left(z_{1}^{\prime}, z_{2}^{\prime}\right)$ и $z^{\prime \prime}=\left(z_{1}^{\prime \prime}, z_{2}^{\prime \prime}\right) \mathrm{c}$ условиями $z^{\prime} \in \beta_{1} \Gamma_{n}, z^{\prime \prime} \in \beta_{2} \Gamma_{n}, \rho\left(z^{\prime}, z^{\prime \prime}\right)<\delta$. Положим

$$
\Delta_{1}=\left[z_{1}^{\prime}, z_{1}^{\prime \prime}\right] \times\left[z_{2}^{\prime}-1, z_{2}^{\prime}\right], \quad c=\omega_{\beta_{1}}^{(n)}\left(x_{0}\right) \omega_{\beta_{2}}^{(n)}\left(x_{0}\right)
$$

где $x_{0} \in \mathbb{S}^{1}$ удовлетворяет условию $x_{0} \Gamma_{n} \notin \Delta_{1}$. Учитывая предложение 1 , имеем

$$
\left\{x \in \mathbb{S}^{1}: \omega_{\beta_{1}}^{(n)}(x) \omega_{\beta_{2}}^{(n)}(x) \neq c\right\} \subseteq\left\{x \in \mathbb{S}^{1}: x \Gamma_{n} \cap \Delta_{1} \neq \varnothing\right\} .
$$

Учитывая неравенства

$$
\begin{aligned}
m\left\{x \in \mathbb{S}^{1}: x \Gamma_{n} \cap \Delta_{1} \neq \varnothing\right\} & \leqslant 2 m\left\{x \in \mathbb{S}^{1}: z_{1}(n, x) \leqslant \delta\right\} \\
& =2 \delta m\left\{x \in \mathbb{S}^{1}: z_{1}(n, x) \leqslant 1\right\}<2 \delta
\end{aligned}
$$

получаем (13).

2. Сдвиг по вертикали на вектор $\bar{a}$. Выберем точки $z^{\prime}=\left(z_{1}^{\prime}, z_{2}^{\prime}\right)$ и $z^{\prime \prime}=\left(z_{1}^{\prime \prime}, z_{2}^{\prime \prime}\right)$ с условиями $z^{\prime} \in \beta_{1} \Gamma_{n}, z^{\prime \prime} \in \beta_{2} \Gamma_{n}, \rho\left(z^{\prime}, z^{\prime \prime}\right)<\delta, z_{2}^{\prime}<z_{2}^{\prime \prime}$. Положим

$$
\Delta_{2}=\left[z_{1}^{\prime}-\frac{b_{n}}{a_{n}}, z_{1}^{\prime}\right] \times\left[z_{2}^{\prime}, z_{2}^{\prime \prime}\right], \quad c=\omega_{\beta_{1}}^{(n)}\left(x_{0}\right) \omega_{\beta_{2}}^{(n)}\left(x_{0}\right)
$$

где точка $x \in \mathbb{S}^{1}$ удовлетворяет условию $x_{0} \Gamma_{n} \notin \Delta_{2}$. Учитывая предложение 1 , имеем

$$
\left\{x \in \mathbb{S}^{1}: \omega_{\beta_{1}}^{(n)}(x) \omega_{\beta_{2}}^{(n)}(x) \neq c\right\} \subseteq\left\{x \in \mathbb{S}^{1}: x \Gamma_{n} \cap \Delta_{2} \neq \varnothing\right\} .
$$


Используя неравенства

$$
\begin{aligned}
m\left\{x \in \mathbb{S}^{1}: x \Gamma_{n} \cap \Delta_{2} \neq \varnothing\right\} \leqslant 2 m\left\{x \in \mathbb{S}^{1}: z_{1}(n, x) \leqslant \frac{b_{n}}{a_{n}}, z_{2}(n, x)<\delta\right\}, \\
\frac{1}{2}\left[\frac{1}{\delta}\right] \cdot m\left\{x \in \mathbb{S}^{1}: z_{1}(n, x) \leqslant 1, z_{2}(n, x)<\delta\right\} \\
\quad<\sum_{k=1}^{[1 / \delta]} m\left\{x \in \mathbb{S}^{1}: z_{1}(n, x) \leqslant 1,(k-1) \delta \leqslant z_{2}(n, x)<k \delta\right\} \\
\leqslant m\left\{x \in \mathbb{S}^{1}: z_{1}(n, x) \leqslant 1\right\}<1,
\end{aligned}
$$

получаем (13). Предложение 2 доказано.

ДокАЗАтЕЛЬство ЛЕммы 6. Ясно, что достаточно доказать лемму для $\beta^{(1)}$ и $\beta^{(2)}$, не совпадающих только на первых $k$ координатах. В силу неравенства треугольника для полуметрики $\rho_{n}^{(k)}$ достаточно доказать лемму для $\beta^{(1)}$ и $\beta^{(2)}$, не совпадающих на одной координате, например на первой или второй. Разберем два соответствующих случая.

1. Пусть $\beta_{12}^{(1)} \neq \beta_{12}^{(2)}$. Если $i_{12}=0\left(\chi=y_{11}^{i_{11}} y_{12}^{i_{12}} \cdots\right)$, то моменты совпадают, иначе имеем

$$
\begin{aligned}
& \left|\operatorname{Mom}_{n}\left(\chi, \beta^{(1)}\right)-\operatorname{Mom}_{n}\left(\chi, \beta^{(2)}\right)\right| \\
& \quad=||\left|\int_{\mathbb{S}^{1}} \omega_{\beta_{11}^{(1)}}^{(n)}\left(x-\beta_{12}^{(1)}\right) \varphi(x) d m(x)\right|-\left|\int_{\mathbb{S}^{1}} \omega_{\beta_{11}^{(1)}}^{(n)}\left(x-\beta_{12}^{(2)}\right) \varphi(x) d m(x)\right| \mid
\end{aligned}
$$

для некоторой функции $\varphi(x): \mathbb{S}^{1} \rightarrow\{-1,1\}$. Используя тождество

$$
\omega_{\beta}(x-\gamma) \equiv \begin{cases}\omega_{\beta+\gamma}(x) \omega_{\gamma}(x) & \text { при } \gamma+\beta \leqslant 1, \\ -\omega_{\beta+\gamma-1}(x) \omega_{\gamma}(x) & \text { при } \gamma+\beta>1,\end{cases}
$$

переписываем правую часть (14) в виде

$$
\begin{aligned}
& || \int_{\mathbb{S}^{1}} \omega_{\beta_{11}^{(1)}+\beta_{12}^{(1)}(\bmod 1)}^{(n)}(x) \omega_{\beta_{12}^{(1)}}^{(n)}(x) \varphi(x) d m(x) \mid \\
& \quad-\left|\int_{\mathbb{S}^{1}} \omega_{\beta_{11}^{(n)}+\beta_{12}^{(2)}(\bmod 1)}^{(x)} \omega_{\beta_{12}^{(2)}}^{(n)}(x) \varphi(x) d m(x)\right| \mid
\end{aligned}
$$

Учитывая предложение 2 при $\varepsilon^{\prime}=\varepsilon / 4$ и инвариантность метрики $\rho_{n}($ см. $\S 2)$ при повороте на $\beta_{11}^{(1)}$, получаем, что две подынтегральные функции в $(15)$, с точностью до умножения на -1 , отличаются на множестве меры менее чем $2 \varepsilon^{\prime}$, т.е. имеем нужную оценку.

2. Пусть $\beta_{11}^{(1)} \neq \beta_{11}^{(2)}$. Без ограничения общности $i_{11}=1$. Имеем

$$
\begin{aligned}
\left|\operatorname{Mom}_{n}\left(\chi, \beta^{(1)}\right)-\operatorname{Mom}_{n}\left(\chi, \beta^{(2)}\right)\right| \\
=||\left|\int_{\mathbb{S}^{1}} \omega_{\beta_{11}^{(1)}}^{(n)}(x) \omega_{\beta_{11}^{(1)}}^{(n)}\left(x-\beta_{12}^{(1)}\right) \cdots \omega_{\beta_{11}^{(1)}}^{(n)}\left(x-\beta_{1 n_{1}}^{(1)}\right) \varphi(x) d m(x)\right| \\
\quad-\left|\int_{\mathbb{S}^{1}} \omega_{\beta_{11}^{(2)}}^{(n)}(x) \omega_{\beta_{11}^{(2)}}^{(n)}\left(x-\beta_{12}^{(1)}\right) \cdots \omega_{\beta_{11}^{(2)}}^{(n)}\left(x-\beta_{1 n_{1}}^{(1)}\right) \varphi(x) d m(x)\right| \mid .
\end{aligned}
$$

Рассуждая, как и при доказательстве пункта 1, получаем требуемую оценку. 


\section{§5. Выбор центров требуемых окрестностей}

Цель этого параграф̆а - по заданной паре характеров $\chi_{1}$ и $\chi_{2}\left(\chi_{1} \nsim \chi_{2}\right)$ и номеру $n$ указать, учитывая $\S 4$, такие элементы $\beta(n)$ из множества $\mathbb{S}^{\widetilde{N}}$, чтобы моменты $\operatorname{Mom}_{n}\left(\chi_{i}, \beta(n)\right), i=1,2$, были равномерно по $n$ отделены друг от друга.

Здесь, как и выше, $\chi_{1}$ и $\chi_{2}$ отождествим с наборами $\left(i_{11}, i_{12}, \ldots\right)$ и $\left(j_{11}, j_{12}, \ldots\right)$.

I. Рассмотрим частный случай $i_{k r}=j_{k r}=0$ при $k=2,3, \ldots, r=1,2, \ldots, n_{k}$. Если неверно равенство

$$
\sum_{r} i_{1 r}=\sum_{r} j_{1 r}(\bmod 2)
$$

то положим $\beta_{12}=\beta_{13}=\cdots=0, \beta_{11}=\beta_{1}$, где $\beta_{1}-$ из леммы 5 . Тогда получим

$$
\operatorname{MoM}_{n}=1-\left|\int_{\mathbb{S} 1} \omega_{\beta_{11}}^{(n)}(x) d m(x)\right| .
$$

Здесь и далее

$$
\operatorname{Mom}_{n}=\left|\operatorname{Mom}_{n}\left(\chi_{1}, \beta(n)\right)-\operatorname{Mom}_{n}\left(\chi_{2}, \beta(n)\right)\right| .
$$

Осталось использовать оценку (10).

Если верно равенство (16), то, учитьвая условие $\chi_{1} \nsim \chi_{2}$, нетрудно убедиться, что с точностью до перемены мест символов $i$ и $j$ имеет место хотя бы один из следующих вариантов:

1. $\exists m_{1}\left(1<m_{1} \& i_{11}=i_{1 m_{1}}=1 \& j_{11}=j_{1 m_{1}}=0 \& \sum_{r} i_{1 r}=0(\bmod 2)\right)$;

2. $\exists m_{1}, m_{2}\left(1<m_{1}<m_{2} \& i_{1 m_{p}}=1 \& j_{1 m_{p}}=0, p=1,2, \& \sum_{r} i_{1 r}=0(\bmod 2)\right)$;

3. $\exists m_{1}, m_{2}\left(1<m_{1}<m_{2} \& i_{1 m_{p}}=1 \& j_{1 m_{p}}=0, p=1,2, \& \sum_{r} i_{1 r}=1(\bmod 2)\right)$;

4. $\exists m_{2}, m_{3}\left(1<m_{p}, p=2,3, \& i_{11}=i_{1 m_{2}}=i_{1 m_{3}}=j_{1 m_{3}}=1 \& j_{11}=j_{1 m_{2}}=0\right.$ $\left.\& \sum_{r} i_{1 r}=1(\bmod 2)\right)$;

5. $\exists m_{1}, m_{2}\left(i_{11}=j_{11}=i_{1 m_{1}}=j_{1 m_{2}}=1 \& j_{1 m_{1}}=i_{1 m_{2}}=0 \& \sum_{r} i_{1 r}=0(\bmod 2)\right)$;

6. $\exists m_{2}, m_{3}\left(i_{11}=i_{1 m_{3}}=j_{1 m_{2}}=j_{1 m_{3}}=1 \& j_{11}=i_{1 m_{2}}=0 \& \sum_{r} i_{1 r}=0(\bmod 2)\right)$;

7. $\exists m_{1}, m_{2}, m_{3}\left(1<m_{p}, p=1,2,3, \& i_{1 m_{1}}=j_{1 m_{2}}=i_{1 m_{3}}=j_{1 m_{3}}=1 \& j_{1 m_{1}}=\right.$ $\left.i_{1 m_{2}}=0 \& \sum_{r} i_{1 r}=0(\bmod 2)\right)$;

8. $\exists m_{1}, m_{2}, m_{3}\left(m_{1} \neq m_{3} \& i_{11}=j_{1 m_{2}}=i_{1 m_{3}}=j_{1 m_{3}}=i_{1 m_{1}}=j_{1 m_{1}}=\right.$ $\left.1 \& j_{11}=i_{1 m_{2}}=0 \& \sum_{r} i_{1 r}=1(\bmod 2)\right)$;

9. $\exists m_{1}, m_{2}, m_{3}\left(m_{3} \neq 1 \& i_{11}=j_{11}=i_{1 m_{1}}=j_{1 m_{2}}=i_{1 m_{3}}=j_{1 m_{3}}=1 \& j_{1 m_{1}}=\right.$ $\left.i_{1 m_{2}}=0 \& \sum_{r} i_{1 r}=1(\bmod 2)\right)$;

10. $\exists m_{1}, m_{2}, m_{3}\left(1<m_{p}, p=1,2,3, \& i_{1 m_{1}}=j_{1 m_{2}}=i_{1 m_{3}}=j_{1 m_{3}}=1 \& j_{1 m_{1}}=\right.$ $\left.i_{1 m_{2}}=0 \& \sum_{r} i_{1 r}=1(\bmod 2)\right)$. 
Если $\exists m_{1}, m_{2}\left(i_{1 m_{p}}=1 \& j_{1 m_{p}}=0, p=1,2\left(m_{1} \neq m_{2}\right)\right)$, то возможен один из вариантов $1-4$. В противном случае для пары $\chi_{1}, \chi_{2}$ подойдет хотя бы один из вариантов $5-10$.

Пусть $\beta_{2}, \ldots, \beta_{6}$ выбраны по лемме 5. Определим ниже $\beta_{1 l}, l=1,2, \ldots, n$, для каждого из случаев $1-10$ :

$1-2$. $\beta_{11}=\beta_{2}, \beta_{1 m_{1}}=\beta_{3}, \beta_{1 l}=0$ при $l \notin\left\{1, m_{1}\right\}$;

3. $\beta_{11}=\beta_{4}, \beta_{1 m_{1}}=\beta_{5}, \beta_{1 m_{2}}=\beta_{6}, \beta_{1 l}=0$ при $l \notin\left\{1, m_{1}, m_{2}\right\}$;

4. $\beta_{11}=\beta_{4}, \beta_{1 m_{2}}=\beta_{5}, \beta_{1 m_{3}}=\beta_{6}, \beta_{1 l}=\beta_{1 m_{3}}$ при $l \notin\left\{1, m_{2}, m_{3}\right\}$;

5. $\beta_{11}=\beta_{2}, \beta_{1 m_{1}}=\beta_{3}, \beta_{1 l}=0$ при $l \notin\left\{1, m_{1}\right\}$;

6-7. $\beta_{11}=\beta_{2}, \beta_{1 m_{2}}=\beta_{3}, \beta_{1 l}=0$ при $l \notin\left\{1, m_{2}\right\}$;

8-10. $\beta_{11}=\beta_{4}, \beta_{1 m_{1}}=\beta_{5}, \beta_{1 m_{3}}=\beta_{6}, \beta_{1 m_{2}}=\beta_{1 m_{3}}, \beta_{1 l}=0$ при $l \notin$ $\left\{1, m_{1}, m_{2}, m_{3}\right\}$;

Отсюда в каждом случае получим, что $\mathrm{Moм}_{n}$ есть в точности соответствующая левая часть одной из формул (11), (12). Следовательно, выбор $\beta(n)$ с заявленными свойствами осуществлен.

II. Общий случай легко следует из I. Действительно, для любого характера $\chi$ введем характеры $\chi(k)$, определяемые наборами $\left(i_{11}^{(k)}, i_{12}^{(k)}, \ldots\right)$, где

$$
i_{l r}^{(k)}= \begin{cases}i_{l r} & \text { при } l=k, \\ 0 & \text { при } l \neq k,\end{cases}
$$

здесь $\chi$ определяется набором $\left(i_{11}, i_{12}, \ldots\right)$ (т.е. $\left.\chi=y_{11}^{i_{11}} y_{12}^{i_{12}} \ldots\right)$.

Если при $\chi_{1} \nsim \chi_{2}$ для некоторого $k_{0}$ верно $\chi_{1}\left(k_{0}\right) \nsim \chi_{2}\left(k_{0}\right)$, то определим $\beta_{k_{0} l}$, $l=1,2, \ldots, n_{k_{0}}$, как и в пункте I, и положим $\beta_{k l}=0$ при $k \neq k_{0}$.

Если для любого $k$ верно $\chi_{1}(k) \sim \chi_{2}(k)$, то тогда можно выбрать $k_{1}, k_{2}, m_{1}$, $m_{2}, m_{3}, m_{4}\left(m_{1} \neq m_{2}, k_{1} \neq k_{2} ; m_{1}, m_{2} \leqslant n_{k_{1}} ; m_{3}, m_{4} \leqslant n_{k_{2}}\right)$ с условиями

$$
\begin{aligned}
& i_{k_{1} r}=1 \Leftrightarrow r=m_{1} ; \quad i_{k_{2} r}=1 \Leftrightarrow r=m_{3} ; \\
& j_{k_{1} r}=1 \Leftrightarrow r=m_{2} ; \quad j_{k_{2} r}=1 \Leftrightarrow r=m_{4}
\end{aligned}
$$

(возможен вариант, когда $m_{3}=m_{4}$ ). Пусть для определенности $m_{1} \neq 1$. Положим $\beta_{k_{1} 1}=\beta_{k_{2} 1}, \beta_{k r}=0$ при $k \notin\left\{k_{1}, k_{2}\right\}, r=1,2, \ldots, n_{k}, \beta_{k_{2} 2}=\beta_{k_{2} 3}=\cdots=$ $\beta_{k_{2} n_{k_{2}}}=0$ и если $m_{2} \neq 1$, то $\beta_{k_{1} m_{2}}=0$. Имеем

$$
\operatorname{Mom}_{n}=1-\left|\int_{\mathbb{S}^{1}} \omega_{\beta_{k_{1} 1}}^{(n)}\left(x-\beta_{k_{1} m_{1}}\right) \omega_{\beta_{k_{1} 1}}^{(n)}(x) d m(x)\right| .
$$

Осталось выбрать $\beta_{k_{1} 1}=\beta_{2}$ и $\beta_{k_{1} m_{1}}=\beta_{3}$, где $\beta_{2}$ и $\beta_{3}-$ из леммы 5 . Выбор $\beta(n)$ в общем случае осуществлен.

\section{§6. Доказательства теорем 2,3, следствий и замечаний}

Этот параграфи использует обозначения $\S \S 2,3$.

6.1. ДокАЗАтЕльство тЕоремы 3. Фиксируем пару характеров $\chi_{1}, \chi_{2} \in \widehat{\mathbb{Z}}_{2}^{\tilde{N}}$ таких, что $\chi_{1} \sim \chi_{2}$. Для определенности пусть $\chi_{1}(y)=y_{11}, \chi_{2}(y)=y_{12}$. Пункт 2 следует из равенства

$$
T_{1-\beta_{12}} V_{\chi_{1}} f(x)=\omega_{\beta_{11}}\left(x-\beta_{12}\right) f\left(x+\alpha-\beta_{12}\right)=V_{\chi_{2}} T_{1-\beta_{12}} f(x) .
$$


Зафиксируем характер $\chi$. Построим плотное подмножество типа $G_{\delta}$ таких $\beta$, для которых $V_{\chi}$ имеет простой спектр. Положим $\beta(n)=(0,0, \ldots), \varepsilon=0.01, k$ определим по характеру (см. лемму 6).

Нетрудно убедиться, что если

$$
d_{n}=\frac{q_{n-1} b_{n}}{q_{n} a_{n}} \geqslant \frac{1}{2}
$$

то $\alpha_{n-1}=\alpha_{n}=1$ и $d_{n+1}<1 / 2$. Пусть

$$
\beta \in \bigcap_{\substack{n>m, d_{n}<\frac{1}{2}}} U_{\varepsilon}(n) .
$$

Фиксируем $n$ с условиями $d_{n}<1 / 2, \beta \in U_{\varepsilon}(n)$ и пусть для определенности $n$ нечетно. Рассмотрим $n$-ю модель (см. рис. 4$)$. Имеем

$$
m\left\{x \in \mathbb{S}^{1}: z_{1}(n, x) \leqslant 1\right\} \geqslant \frac{2}{3} .
$$

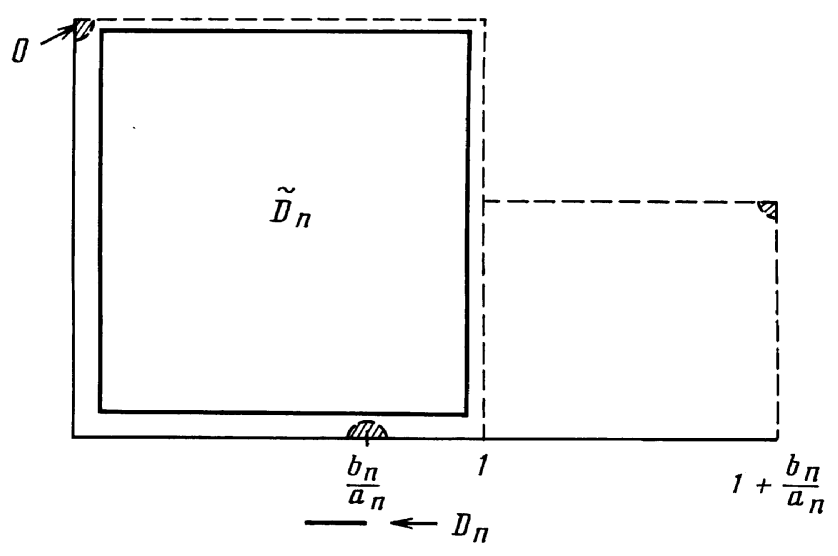

Рис. 4

Функция $\omega_{\chi}(x)$ может иметь разрывы только в точках $\beta_{i j}, \beta_{i j}+\beta_{i 1}$, где $\beta=$ $\left(\beta_{11}, \beta_{12}, \ldots\right)$. Учитывая инвариантность метрики $\rho_{n}$ относительно поворота, имеem

$$
\rho_{n}\left(\beta_{i j}, 0\right)<0.01, \quad \rho_{n}\left(\beta_{i j}+\beta_{i 1}, 0\right)<0.02
$$

для таких $\beta_{i j}$, от которых зависит $\omega_{\chi}(x)$. Заметим, что $z_{1}(n, 0)=0$ и $z_{2}(n, 0)=$ $1-1 / q_{n}$. Следовательно, все точки разрыва функции $\omega_{\chi}(x)$ в $n$-й модели расположены в заштрихованных зонах на рис. 4 .

Положим $h_{n}(x)=\chi_{D_{n}}^{\prime}, \widetilde{D}_{n}=\bigcup_{j=0}^{\left[0.98 q_{n}-1\right]} T_{\alpha}^{-j} D_{n}$, где $\chi_{A}^{\prime}$-индикатор множества $A, D_{n}=\left\{x \in \mathbb{S}^{1}: z_{2}(n, x)=z_{2}(n, 0), 0.02<z_{1}(n, x)<0.98\right\}$. Учитьвая (18), при достаточно большом $n$ имеем

$$
m\left(\widetilde{D}_{n}\right) \geqslant 0.6
$$


Так как $\left\|\chi_{\widetilde{D}_{n}}^{\prime}-T_{\alpha} \chi_{\widetilde{D}_{n}}^{\prime}\right\| \rightarrow 0$ при $n \rightarrow \infty$ и $T_{\alpha}-$ эргодично, то по лемме 4 для любой $f$ из $L_{2}\left(\mathbb{S}^{1}, m\right)$ имеем

$$
\int_{\widetilde{D}_{n}} f d m-m\left(\widetilde{D}_{n}\right) \int_{\mathbb{S}^{1}} f d m \rightarrow 0 \text { при } n \rightarrow \infty .
$$

Используем следующий хорошо известный классический факт.

ПРЕДЛОЖЕНИЕ 3. Если существуют $\theta>0$ и последовательность векторов $h_{n}$ из $L_{2}\left(\mathbb{S}^{1}, m\right)$, обладающие свойством

$$
\forall h \quad(\|h\|=1) \quad \varlimsup_{n \rightarrow \infty} d^{2}\left(C_{U}\left(h_{n}\right), h\right) \leqslant \frac{1}{2}-\theta,
$$

то унитарньй оператор $U$ имеет простой спектр, әде $C_{U}(f)$ - ииклическое пространство вектора $f$ относительно $U$,

$$
d\left(g, C_{U}(h)\right)=\min _{f \in C_{U}(h)}\|g-f\|_{L_{2}} .
$$

Свойство (21) достаточно доказать для всюду плотного подмножества векторов $h$. Поэтому рассмотрим только функцию $h$, постоянную на всех полуинтервалах в $k$-й модели, начиная с некоторого $k$. Без ограничения общности $k \leqslant n$. Тогда $h(x)$ и $\omega_{\chi}(x)$ постоянны на интервалах $T_{\alpha}^{-j} D_{n}, j=0,1, \ldots,\left[0.98 q_{n}\right]-1$. Следовательно, $V_{\chi}^{l} \chi_{D_{n}}^{\prime}=d(l) \chi_{T_{\alpha}^{-1} D_{n}}^{\prime}$, где $l=0,1, \ldots,\left[0.98 q_{n}\right]-1$, a $d(l)$ не зависит от $x$. Отсюда $\chi_{T_{\alpha}^{-l} D_{n}}^{\prime} \in C_{V_{\chi}}\left(h_{n}\right)$. Поэтому

$$
h(x) \chi_{\widetilde{D}_{n}}^{\prime}=\sum_{j} h(x) \cdot \chi_{T_{\alpha}^{-j} D_{n}}^{\prime} \in C_{V_{\chi}}\left(h_{n}\right)
$$

Следовательно, учитывая (19) и (20), имеем

$$
\begin{aligned}
d^{2}\left(C\left(h_{n}\right), h\right) & \leqslant\left\|h \cdot \chi_{\widetilde{D}_{n}}^{\prime}-h\right\|^{2}=\int_{\mathbb{S}^{1} \backslash \widetilde{D}_{n}}|h|^{2} d m \\
& =\left(1-m\left(\widetilde{D}_{n}\right)\right)+\left(m\left(\widetilde{D}_{n}\right) \cdot \int_{\mathbb{S}^{1}}|h|^{2} d m-\int_{\widetilde{D}_{n}}|h|^{2} d m\right)<0.41
\end{aligned}
$$

при достаточно большом $n$. Осталось учесть предложение 3 и топологическую лемму для множества в формуле (17).

Фиксируем пару $\chi_{1}, \chi_{2}$ такую, что $\chi_{1} \nsim \chi_{2}$. Определим $\beta(n), \delta>0$, номер $k$, а значит, и $U_{\delta}(n)$. Для любого $n$ и выбранных в $\S 5 \beta(n)$ имеем

$$
\left|\operatorname{Mom}_{n}\left(\chi_{1}, \beta(n)\right)-\operatorname{Mom}_{n}\left(\chi_{2}, \beta(n)\right)\right|>\varepsilon,
$$

где $\varepsilon$ определено в лемме 5 . Выберем такие $\delta>0$ и номер $k$ (см. лемму 6 ), что для любых $n, i=1,2$ и $\beta^{(1)}, \beta^{(2)} \in \mathbb{S}^{\widetilde{N}}$

$$
\rho_{n}^{(k)}\left(\beta^{(1)}, \beta^{(2)}\right)<\delta \Rightarrow\left|\operatorname{Mom}_{n}\left(\chi_{i}, \beta^{(1)}\right)-\operatorname{Mom}_{n}\left(\chi_{i}, \beta^{(2)}\right)\right|<\frac{\varepsilon}{3} .
$$


Пусть $\beta \in \bigcap_{m} \bigcup_{n>m} U_{\delta}(n)$, тогда $\beta \in \bigcap_{l} U_{\delta}\left(n_{l}^{\prime}\right)$ для некоторой последовательности $n_{l}^{\prime} \rightarrow \infty$ при $l \rightarrow \infty$. Следовательно, $\rho_{n_{l}^{\prime}}^{(k)}\left(\beta, \beta\left(n_{l}^{\prime}\right)\right)<\delta$ для любого $l$. Учитывая (22), (23), для любого $l$ получаем

$$
\left|\operatorname{Mom}_{n_{l}^{\prime}}\left(\chi_{1}, \beta\right)-\operatorname{Mom}_{n_{l}^{\prime}}\left(\chi_{2}, \beta\right)\right|>\frac{\varepsilon}{3} .
$$

Выбирая (если потребуется) подпоследовательность $n_{l_{p}}^{\prime}$, учитывая лемму 2, получаем

$$
V_{\chi_{i}}^{q_{n^{\prime}}^{\prime}} \rightarrow c_{i} \cdot E \quad \text { при } \quad p \rightarrow \infty, \quad i=1,2
$$

В силу (24) имеем

$$
\left|c_{1}-c_{2}\right|>\frac{\varepsilon}{3}
$$

Осталось применить лемму 3 и топологическую лемму.

Искомьм плотным подмножеством типа $G_{\delta}$ будет пересечение плотных подмножеств типа $G_{\delta}$, введенных выше. Теорема 2 доказана.

6.2. ДоКАЗАТЕЛЬство СЛЕДствИя 1. Если преобразование $T$ эргодично, то, очевидно, $1 \in \mathscr{M}(T)$. Следовательно, достаточно так надстроить любое преобразование $T_{\omega_{\beta}}$ из теоремы 1 , чтобы у функции кратности спектра добавилось только значение $\infty$.

Рассмотрим декартово произведение $\widetilde{T}=T_{\omega_{\beta}} \times T_{0}$, где $\mathscr{M}\left(T_{0}\right)=\{1, \infty\}$ и компонента кратности 1 состоит из констант (например, $T_{0}$ - автоморфизм Бернулли).

Преобразование $\widetilde{T}$ эргодично в силу непрерывности спектра $T_{0}$.

Гильбертово пространство $L_{2}\left(\mathbb{S}^{1} \times\{-1,1\}^{\widetilde{N}} \times X, m \times l^{\widetilde{N}} \times \mu\right)$ представимо в виде прямой суммы инвариантных относительно $\widetilde{T}$ подпространств $H_{i}, i \in \mathbb{Z}_{+}$, где $H_{i}$ - наименьшее замкнутое подпространство, содержащее конечные линейные комбинации функций вида $f(x, y) \cdot \psi(z)$, где $f(x, y) \in L_{2}\left(\mathbb{S}^{1} \times\{-1,1\}^{\widetilde{N}}, m \times l^{\widetilde{N}}\right)$, $\psi(z) \in C\left(h_{i}\right), C\left(h_{i}\right)$ - $i$-е циклическое пространство в спектральном разложении оператора $T_{0}\left(C\left(h_{0}\right)=\mathbb{C} \cdot \mathbf{1}\right)$. Осталось заметить, что

$$
\begin{aligned}
\left.\widetilde{T}\right|_{H_{0}} & \left.\sim T_{\omega_{\beta}}\right|_{L_{2}\left(\mathbb{S}^{1} \times\{-1,1\}^{\widetilde{N}}, m \times l^{\widetilde{N}}\right)} \\
\left.\widetilde{T}\right|_{H_{j}} & \left.\sim \widetilde{T}\right|_{H_{i}} \text { при } 0 \neq i \neq j \neq 0,
\end{aligned}
$$

где “〜” - спектральный изоморфиизм.

6.3. ДокАЗАТЕЛьСтво слЕДствия 2. По теореме 1 преобразование $T_{\omega_{\beta}}$ с заданной ограниченной функцией кратности спектра может действовать в пространстве $\mathbb{S}^{1} \times\{-1,1\}^{\widetilde{N}}$, состоящем из $2^{\widetilde{N}}$ полуинтервалов (для некоторого $\left.\widetilde{N}<\infty\right)$. Расположим на оси друг за другом эти полуинтервалы. В силу того, что функция $\omega_{\beta}(x)$ кусочно постоянна, преобразование $T_{\omega_{\beta}}$ есть перекладьвание на полученном полуинтервале. Доказательство следствия 2 окончено.

6.4. ДоКАЗАТЕЛЬСТво ЗАмЕчАния 1. Достаточно для каждого характера $\chi \in \widehat{\mathbb{Z}}_{2}^{\widetilde{N}} \backslash\{\mathbf{1}\}$ построить плотное подмножество типа $G_{\delta}$ таких $\beta$, для которых оператор $V_{\chi}$ имеет непрерывный спектр. Используем следующее простое наблюдение, не нуждающееся в доказательстве. 
ПРЕДЛОЖЕНИЕ 4. Если для некоторого $с \in \mathbb{C},|c|<1$, последовательность операторов $V_{\chi}^{k_{m}}$ слабо сходится $\kappa$ cE при $m \rightarrow \infty$, то унитарный оператор $V_{\chi}$ не имеет собственных векторов.

Зафиксируем номер $n$ и такое $r_{0}$, что $i_{r_{0} j_{0}}=1$ для некоторого $j_{0} \leqslant n_{r_{0}}$ $\left(\chi=y_{11}^{i_{11}} y_{12}^{i_{12}} \cdots\right)$. Определим $\beta(n)=\left(\beta_{11}, \beta_{12}, \ldots\right) \in \mathbb{S}^{\tilde{N}}, \delta>0, k$. При $r \neq r_{0}$ положим $\beta_{r j}=0$. Если

$$
\sum_{j} i_{r_{0} j}=1(\bmod 2)
$$

то пусть

$$
\beta_{r_{0} j}=\left\{\begin{array}{l}
\beta_{1} \text { при } j=1, \\
0 \quad \text { иначе }
\end{array}\right.
$$

где $\beta_{1}$ выбрано по лемме 5. Если не верно (25), то существует такое $j_{1}, j_{1} \leqslant n_{r_{0}}$, что $j_{0} \neq j_{1}$ и $i_{r_{0} j_{1}}=1$. В этом случае

$$
\beta_{r_{0} j}=\left\{\begin{array}{l}
\beta_{2} \text { при } j=1, \\
\beta_{3} \text { при } j=j_{t_{0}} \\
0 \quad \text { иначе }
\end{array}\right.
$$

где $t_{0} \in\{0,1\}$ выбрано с условием $j_{t_{0}} \neq 1$, а $\beta_{2}, \beta_{3}$ выбраны по лемме 5 .

Учитьвая $(10),(11)$ соответственно, для любого $n$ имеем

$$
\operatorname{Mom}_{n}(\chi, \beta(n))<1-\varepsilon
$$

В силу леммы 6 выберем такие $\delta>0$ и $k$ ( $\chi$ уже задан), что неравенство $\rho_{n}^{(k)}\left(\beta^{(1)}, \beta^{(2)}\right)<\delta$ влечет

$$
\left|\operatorname{Mom}_{n}\left(\chi, \beta^{(1)}\right)-\operatorname{Mom}_{n}\left(\chi, \beta^{(2)}\right)\right|<\frac{\varepsilon}{2}
$$

Пусть $\beta \in \bigcap_{m} \bigcup_{n>m} U_{\delta}(n)$, тогда $\beta \in \bigcap_{l} U_{\delta}\left(n_{l}^{\prime}\right)$ для некоторой последовательности $n_{l}^{\prime}$ с условием $n_{l}^{\prime} \rightarrow \infty$ при $l \rightarrow \infty$.

Следовательно, $\rho_{n_{l}^{\prime}}^{(k)}\left(\beta, \beta\left(n_{l}^{\prime}\right)\right)<\delta$ для любого $l$. Учитывая $(26),(27)$, для любого $l$ получаем

$$
\operatorname{MoM}_{n_{l}^{\prime}}(\chi, \beta)<1-\frac{\varepsilon}{2}
$$

Выбираем (если потребуется) подпоследовательность $n_{l_{p}}^{\prime}$, имеем

$$
\operatorname{Mom}_{n_{l_{p}}^{\prime}}(\chi, \beta) \rightarrow c<1 \text { при } p \rightarrow \infty
$$

Для завершения доказательства достаточно последовательно применить лемму 2 для операторов $V_{\chi}^{q_{n_{p}^{\prime}}^{\prime}}$, предложение 4 и топологическую лемму. 
6.5. ДоКАЗАТЕЛЬСТВо ЗАМЕчАнИЯ 2. Учитьвая структуру построенного в теореме 3 множества, замечание, очевидно, следует из следующего утверждения: $\forall \delta>0 \forall k \forall r \forall n_{l}^{\prime}, l=1,2, \ldots$,

$$
\lim _{l \rightarrow \infty} n_{l}^{\prime}=+\infty \Rightarrow m \times m \times \cdots\left(\bigcup_{l>r} U_{\delta}\left(n_{l}^{\prime}\right)\right)=1
$$

Докажем (28). Преобразования

$$
T_{i}=\underbrace{E \times E \times \cdots \times E}_{i-1 \text { раз }} \times T_{\alpha} \times \underbrace{E \times \cdots}_{\tilde{N}-i \text { раз }}
$$

действуют в $\mathbb{S}^{\widetilde{N}}$ и как операторы в $L_{2}\left(\mathbb{S}^{\tilde{N}}, m \times m \times \cdots\right)$. Рассматривая $n_{l}^{\prime}$-е модели, получаем

$$
\begin{gathered}
\exists c>0 \quad \forall l \quad \int_{\mathbb{S}^{N}} f_{l} d(m \times m \times \cdots)>c, \\
\left\|T_{i} f_{l}-f_{l}\right\|_{L_{2}} \rightarrow 0 \quad \forall i \quad \text { при } l \rightarrow \infty,
\end{gathered}
$$

здесь и далее $f_{l}$ - индикатор множества $U_{\delta}\left(n_{l}^{\prime}\right)$. Используя лемму 4 , получаем

$$
f_{l}-\operatorname{Pr}_{T_{i}} f_{l} \rightarrow 0 \quad \forall i \text { при } l \rightarrow \infty
$$

$(\rightarrow-$ слабая сходимость $)$. Если $g_{n} \rightarrow 0$ при $n \rightarrow \infty$, то, очевидно, $\operatorname{Pr}_{T_{i}} g_{n} \rightarrow 0$ для любого $i$ при $n \rightarrow \infty$, и ввиду (30) получим

$$
f_{l}-P_{0} f_{l} \rightarrow 0 \text { при } l \rightarrow \infty
$$

где $P_{0}=\operatorname{Pr}_{T_{1}} \operatorname{Pr}_{T_{2}} \ldots \operatorname{Pr}_{T_{k}}$ - проектор на подпространство функций, не зависящих от первых $k$ координат пространства $\mathbb{S}^{\widetilde{N}}$. Но $f_{l}$ зависит только от первых $k$ координат. Поэтому

$$
P_{0} f_{l}=\int_{\mathbb{S}^{N}} f_{l} d(m \times m \times \cdots) \cdot \mathbf{1}
$$

Перепишем (31) в виде

$$
\int_{\mathbb{S} \widetilde{N}} f_{l} \bar{g} d(m \times \cdots)-\int_{\mathbb{S} \widetilde{N}} f_{l} d(m \times \cdots) \cdot \int_{\mathbb{S} \widetilde{N}} \bar{g} d(m \times \cdots) \rightarrow 0
$$

$\forall g \in L_{2}$ при $l \rightarrow \infty$. Рассматривая в качестве $g$ индикатор множества $\bigcup_{l>r} U_{\delta}\left(n_{l}^{\prime}\right)$, учитывая (29), получаем (28).

6.6. ДокАЗАТЕЛЬСТво ЗАмЕчАния 3. Предположим противное. Тогда существует инвариантная функция в некотором $H_{\chi_{0}}$, где $\chi_{0} \not \equiv \mathbf{1}$, т.е. функция вида $\varphi(x) \chi_{0}(y)$, где $\varphi(x+\alpha) \omega_{\chi_{0}}(x) \chi_{0}(y)=\varphi(x) \chi_{0}(y)$ для почти всех $(x, y) \in$ $\mathbb{S}^{1} \times\{-1,1\}^{\widetilde{N}}$. Отсюда $|\varphi(x)|$ инвариантна относительно эргодического $T_{\alpha}$, поэтому $|\varphi(x)|=$ const для почти всех $x$. Можно считать, что $|\varphi(x)|=1$. Тогда оператор

$$
U: f(x, y) \mapsto \varphi(x) \chi_{0}(y) f(x, y)
$$


унитарен. Заметим, что для $f \in L_{2}\left(\mathbb{S}^{1}, m\right), \chi(y) \in \widehat{\mathbb{Z}}_{2}^{\widetilde{N}}$

$$
\begin{aligned}
& T_{\omega_{\beta}} U f(x) \chi(y)=\varphi(x+\alpha) f(x+\alpha) \omega_{\chi_{0} \chi}(x) \chi_{0}(y) \chi(y), \\
& U T_{\omega_{\beta}} f(x) \chi(y)=\varphi(x) f(x+\alpha) \omega_{\chi}(x) \chi_{0}(y) \chi(y) .
\end{aligned}
$$

Учитывая равенство $\omega_{\chi_{0} \chi}(x)=\omega_{\chi_{0}}(x) \omega_{\chi}(x)$, получаем

$$
U T_{\omega_{\beta}}=T_{\omega_{\beta}} U
$$

Заметим, что оператор $U$ переставляет пространства $H_{\chi}$ как инволюция и

$$
\left.\left.T_{\omega_{\beta}}\right|_{H_{\chi}} \sim T_{\omega_{\beta}}\right|_{U H_{\chi}}
$$

для любого $\chi$. Поэтому функция кратности спектра $T_{\omega_{\beta}}$ принимает только четные значения. Полученное противоречие доказывает замечание 3.

6.7. ДоКАЗАТЕЛЬСТво ТЕОРЕМЫ 2. Равенство $\mathscr{M}\left(T_{\omega_{\beta}}\right)=\mathscr{M}$ и эргодичность $T_{\omega_{\beta}}$ для почти всех $\beta$ следуют из замечаний 2 и 3.

Преобразование $T_{\omega_{\beta}}$ является косым сдвигом на коммутативной компактной групше $\mathbb{S}^{1} \times \widehat{\mathbb{Z}}_{2}^{\widetilde{N}}$. Поэтому из эргодичности $T_{\omega_{\beta}}$ при $\tilde{N}<\infty$ следует строгая эргодичность (см. [21]). Доказательство теоремы 2 окончено.

Заметим, что при $\widetilde{N}=\infty$ эргодические преобразования $T_{\omega_{\beta}}$ также являются строго эргодическими, поскольку являются пределом расширяющейся последовательности строго эргодических факторов.

\section{§7. Конструктивные примеры (типичные точки)}

7.1. Мотив. Информация о типичности некоторого множества еще не содержит указания на то, какими конкретно являются точки из этого множества. Далее будем строить такие типичные точки, которые тесно связаны с типичными точками некоторых одномерных отображений или композиций таковых.

7.2. Редукция к одномерам. Для наглядности рассмотрим $\alpha=[1,1, \ldots]=$ $(\sqrt{5}-1) / 2$ и $\widetilde{N}<\infty$ (разбор остальных случаев проводится по той же схеме с очевидными изменениями). Учитывая (4) и формулу

$$
\frac{q_{n-1}}{q_{n}}=\frac{1}{\alpha_{n-1}+\frac{1}{\alpha_{n-2}+\cdots+\frac{1}{\alpha_{2}+\frac{1}{\alpha_{1}}}}},
$$

имеем

$$
\lim _{n \rightarrow \infty} \frac{q_{n-1}}{q_{n}}=\frac{\sqrt{5}-1}{2}=\frac{b_{n}}{a_{n}} .
$$

Поэтому

$$
\lim _{n \rightarrow \infty} d_{n}=\lim _{n \rightarrow \infty} \frac{q_{n-1} b_{n}}{q_{n} a_{n}}=\frac{3-\sqrt{5}}{2}<\frac{1}{2} .
$$

Рассмотрим $n$-ю модель, $n=2,3, \ldots$ По нечетным $n$ множества $D(n)$ "стабилизируются" к $D_{1} \subseteq \mathbb{R}^{2}$ (по четным - к $D_{0}$ ). Ясно, что точки $\beta_{i}=\beta_{i}(n) \in \mathbb{S}^{1}$, 
$i=1,2, \ldots, 6$, из леммы 5 можно выбрать стабилизирующимися в том смысле, что начиная с некоторого $n_{0}$ координаты $z_{j}\left(n, \beta_{i}(n)\right)$ зависят только от четности $n . \mathrm{B}$ силу (32) в теореме 3 искомое типичное множество есть пересечение не более чем счетного числа множеств вида

$$
\bigcap_{m} \bigcup_{n>m} U_{\varepsilon}(n) \quad(\varepsilon>0),
$$

где центры $\beta(n) \in \mathbb{S}^{\widetilde{N}}$ окрестностей $U_{\varepsilon}(n)($ см. $\S \S 5,6)$ также стабилизируются (в действительности, при $\widetilde{N}<\infty$ имеем пересечение конечного числа множеств вида (33)).

$$
\begin{aligned}
& \text { Для } \beta=\left(\beta_{11}, \beta_{12}, \ldots\right) \in \mathbb{S}^{\widetilde{N}} \text { обозначим } \\
& \qquad \widetilde{\psi}_{n}(\beta)=\left(\psi_{n}\left(\beta_{11}\right), \psi_{n}\left(\beta_{12}\right), \ldots\right) \in D(n) \times D(n) \times \cdots,
\end{aligned}
$$

где $\psi_{n}=\varphi_{n-1} \varphi_{n-2} \ldots \varphi_{1}$ и $\varphi_{i}$ из $\S 2$. Ясно, что если орбита точки $\beta$ всюду плотна в $\widetilde{D}_{0}=D_{0} \times D_{0} \times \cdots$, а именно, $\left\{\widetilde{\psi}_{n}(\beta)\right\}_{n \in 2 \mathbb{N}}$ всюду плотно в $\widetilde{D}_{0}$, то $\beta$ будет типичной, т.е. будет содержаться в каждом множестве (33).

Фиксируем окрестность $U \subseteq D_{0} \subseteq \mathbb{R}^{2}$. Рассмотрим множества

$$
\varphi_{n-1}^{-1}(U \cap D(n)), \varphi_{n-2}^{-1} \varphi_{n-1}^{-1}(U \cap D(n)), \ldots, \varphi_{k}^{-1} \varphi_{k+1}^{-1} \cdots \varphi_{n-1}^{-1}(U \cap D(n))
$$

при достаточно большом $n, 1<k<n$. Очевидно, что локально $\varphi_{p}^{-1}$ растягивает вертикальные слои, а горизонтальные - сжимает. Следовательно, при любом $k$, меньшем некоторого $k_{0}$, сушествует такой интервал $\Delta_{k} \subseteq\left[0,1+b_{k} / a_{k}\right)=$ $[0,(\sqrt{5}+1) / 2)$, что

$$
\varphi_{n-1} \varphi_{n-2} \ldots \varphi_{k}\left\{\left(z_{1}, z_{2}\right) \in D(k): z_{1} \in \Delta_{k}\right\} \subseteq U
$$

Поэтому если последовательность $\left\{\left(z_{1}\left(n, \beta_{11}\right), z_{1}\left(n, \beta_{12}\right), \ldots\right)\right\}_{n \in 2 \mathbb{N}}$ всюду плотна в $[0,(\sqrt{5}+1) / 2)^{\tilde{N}}$, то орбита точки $\beta=\left(\beta_{11}, \beta_{12}, \ldots\right)$ всюду плотна в $\widetilde{D}_{0}$.

Ограничение отображения $\varphi_{2 n+1} \varphi_{2 n}$ на первую координату дает одномерное отображение на $[0,(\sqrt{5}+1) / 2)$ следующего вида

$$
f(z)= \begin{cases}\frac{z}{\alpha^{2}}, & 0 \leqslant z<\alpha \\ \frac{z-\alpha}{\alpha^{2}}, & \alpha \leqslant z<2 \alpha \\ \frac{z-\alpha}{\alpha^{2}}-1, & 2 \alpha \leqslant z<1+\alpha\end{cases}
$$

где $\alpha=(\sqrt{5}-1) / 2$. Определим действие эндоморфизма $T$ на $[0,1)$ по формуле

$$
T z= \begin{cases}\frac{z}{\alpha^{2}}, & 0 \leqslant z<\alpha^{2}, \\ \frac{z}{\alpha^{2}}-1, & \alpha^{2} \leqslant z<2 \alpha^{2}, \\ \frac{z-1}{\alpha^{2}}+1, & 2 \alpha^{2} \leqslant z<1\end{cases}
$$




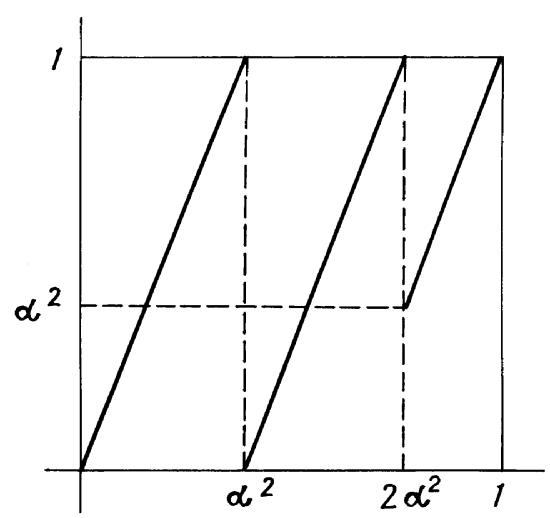

Рис. 5

(см. рис. 5). Так как $T=a f a^{-1}$, где $a(z)=(\sqrt{5}+1) z / 2$, то последовательность $\left\{\left(z_{1}\left(n, \beta_{11}\right), z_{1}\left(n, \beta_{12}\right), \ldots\right)\right\}_{n \in 2 \mathbb{N}}$ всюду плотна в точности тогда, когда

$$
\bigcup_{i \in \mathbb{N}}\left(T^{i}\left(\alpha z_{1}\left(2, \beta_{11}\right)\right), T^{i}\left(\alpha z_{1}\left(2, \beta_{12}\right)\right), \ldots\right) \text { всюду плотно в }[0,1)^{\widetilde{N}} .
$$

Перейдем к символической реализации $T$. Разбиение $\eta=\left\{\left[0, \alpha^{2}\right),\left[\alpha^{2}, 2 \alpha^{2}\right)\right.$, $\left.\left[2 \alpha^{2}, 1\right)\right\}$ является образующим. Пара $(\eta, T)$ естественньм образом задает стационарный случайный процесс $T^{\prime}$ в пространстве $\Omega \subseteq\{0,1,2\}^{\mathbb{N}}$, состоящем из всех допустимых последовательностей, т.е. последовательностей, не содержащих комбинацию "20". Нетрудно по точке $x \in \Omega$ конструктивно определить ее прообраз $\psi^{-1}(x) \in[0,1)$. Ясно, что $(34)$ имеет место в точности тогда, когда множество

$$
\bigcup_{i}\left({T^{\prime}}^{i} \psi\left(\alpha z_{1}\left(2, \beta_{11}\right)\right),{T^{\prime}}^{i} \psi\left(\alpha z_{1}\left(2, \beta_{12}\right)\right), \ldots\right)
$$

всюду плотно в $\Omega^{\widetilde{N}}$. Следовательно, достаточно указать набор точек $\left\{e^{(i)}\right\}_{i=1}^{\widetilde{N}} \subseteq$ $\Omega$ со следуюшим свойством: для любого $n$ и любых допустимых наборов чисел $x_{1}^{(i)}, x_{2}^{(i)}, \ldots, x_{n}^{(i)}$, где $x_{j}^{(i)} \in\{0,1,2\}$, сушествует такое $k$, что $e_{j+k}^{(i)}=x_{j}^{(i)}, j=$ $1,2 \ldots, n, i=1,2, \ldots, \widetilde{N}, e^{(i)}=\left(e_{1}^{(i)}, e_{2}^{(i)}, \ldots\right)$.

Обозначим через $B_{i}^{(j)}$ все допустимые наборы длины $i, i \geqslant 2, j=1,2, \ldots, k_{i}$, занумерованные так, что набор $B_{i}^{(1)} B_{i}^{(2)} \ldots B_{i}^{\left(k_{i}\right)} B_{i}^{(1)}$ тоже будет допустимым. Положим

$$
\begin{aligned}
C_{i}^{(j)} & =\underbrace{B_{i}^{(1)} B_{i}^{(1)} \ldots B_{i}^{(1)}}_{k_{i}^{j-1} \text { раз }} \underbrace{B_{i}^{(2)} \ldots B_{i}^{(2)}}_{k_{i}^{j-1} \text { раз }} \cdots \underbrace{B_{i}^{\left(k_{i}\right)} \ldots B_{i}^{\left(k_{i}\right)}}_{k_{i}^{j-1} \text { раз }}, \\
\widetilde{C}_{i}^{(j)} & =\underbrace{C_{i}^{(j)} \ldots C_{i}^{(j)}}_{k_{i}^{\widetilde{N}-j} \text { раз }}, \\
e^{(j)} & =\left(\widetilde{C}_{2}^{(j)}, 1, \widetilde{C}_{3}^{(j)}, 1, \ldots\right), \quad j=1,2, \ldots, \widetilde{N} .
\end{aligned}
$$

Ясно, что точки $e^{(j)}$ искомые. Подытоживая $\S 7$ с учетом (5), заключаем, что имеет место следующее 
ПРЕДЛОЖЕНИЕ 5. Пусть $\alpha=(\sqrt{5}-1) / 2, \widetilde{N}<\infty, e^{(j)}$ выбраны в $(35), \beta_{j}=$ $\psi^{-1}\left(e^{(j)}\right), \beta=\left(\beta_{1}, \beta_{2}, \ldots, \beta_{\widetilde{N}}\right)$. Тогда преобразование $T_{\omega_{\beta}}$ из $(2)$ әргодично и $\mathscr{M}\left(T_{\omega_{\beta}}\right)=\mathscr{M}$.

Ясно, что построенные перекладывания являются строго эргодическими (см. доказательство теоремы 2).

В заключение отметим, что возможно прямое построение типичной точки $\beta$ при использовании добавок на $n$-м шаге, соответствующих геометрически изменению только координат $z_{1}\left(n, \beta_{i j}\right)$ в $n$-й модели, однако этот путь значительно больше загроможден техническими деталями.

\section{Список литературы}

1. Каток А. Б., Синай Я. Г., Стёпин А. М. Теория динамических систем и общих групп преобразований с инвариантной мерой // Итоги науки и техники. Матем. анализ. Т. 13. М.: ВИНИТИ, 1975. С. 129-262.

2. Вериик A.M., Юзвинский C.A. Динамические системы с инвариантной мерой // Итоги науки и техники. Матем. анализ. М.: ВИНИТИ, 1969. С. 133-187.

3. Neumann J. von. Zur Operatorenmethode in der Klassischen Mechanik // Ann. of Math. (2). 1932. V. 33. P. 587-642.

4. Рохлин В. А. Лекции по энтропийной теории преобразований с инвариантной мерой // УМH. 1967. Т. 22. № 5. С. 3-56.

5. Вершик А.М., Корнфельд И.П., Синай Я.Г. Общая эргодическая теория групा преобразований с инвариантной мерой // Итоги науки и техники. Совр. проблемы матем. Фундамент. направления. Т. 2. М.: ВИНИТИ, 1985. С. 5-111.

6. Агеев O.H. Динамические системы с четнократной лебеговской компонентой в спектре // Матем. сб. 1988. Т. 136 (178). С. 307-319.

7. Lemanczyk $M$. Toeplitz $\mathbb{Z}_{2}$-extensions // Ann. Inst. H. Poincaré Probab. Statist. 1988. V. 24. № 1. P. 1-43.

8. Корнфельд И. П., Синай Я. Г., Фомин С. В. Эргодическая теория. М.: Наука, 1980.

9. Оселедец В.Н. О спектре эргодических автоморфизмов // Докл. АН СССР. 1966. T. 168. № 5. C. 1009-1011.

10. Robinson E. A. Ergodic measure preserving transformations with arbitrary finite spectral multiplicities // Invent. Math. 1983. V. 72. № 2. P. 299-314.

11. Goodson G.R. On the spectral multiplicity of a class of finite rank transformations // Proc. Amer. Math. Soc. 1985. V. 93. № 2. P. 303-306.

12. Robinson E. A. Transformations with highly nonhomogeneous spectrum of finite multiplicity // Israel J. Math. 1986. V. 56. № 1. P. 75-78.

13. Goodson G. R., Kwiatkowski J., Lemanczyk M., Liardet P. On the multiplicity function of ergodic groupextensions of rotations // Studia Math. 1992. V. 102. № 2. P. 157-174.

14. Blanchard F., Lemanczyk $M$. Measure-preserving diffeomorphisms with an arbitrary spectral multiplicity // Topol. Methods Nonlinear Anal. 1993. V. 1. № 2. P. 275-294.

15. Агеев $O . H$. Перемешивание в компонентах и перекладывания $T_{\alpha, \beta} / /$ УМН. 1994. Т. 49. № 2. C. 143-144.

16. Агеев О.Н. Спектральнњй тип перекладьваний $T_{\alpha, \beta} / /$ Матем. сб. 1997. Т. 188. № 8 . C. $13-44$

17. Veech W. A. The metrical theory of interval exchanges transformations. I. General spectral properties // Amer. J. Math. 1984. V. 106. P. 1331-1359.

18. Katok A. B. Constructions in ergodic theory // Preprint, 1986.

19. Каток A.Б., Стёпин A.M. Аппроксимации в эргодической теории // УМН. 1967. T. 22. № 5. C. 81-106.

20. Keane $M$. Nonergodic interval exchange transformations // Israel J. Math. 1977. V. 26. № 2. P. $188-196$.

21. Fürstenberg H. Strict ergodicity and transformation of the torus // Amer. J. Math. 1961. V. 83. № 4. P. 573-601.

Московский государственнњй

технический университет им. Н.Э. Баумана

Поступила в редакцию 20.02.1998 